\title{
Multistability in a quasiperiodically forced piecewise smooth dynamical system
}

\author{
Gaolei Li ${ }^{a, b} \quad$ Yuan Yue $^{a, b} 1$ Jianhua Xie ${ }^{a}$ Celso Grebogi ${ }^{c}$ \\ ${ }^{a}$ School of Mechanics and Engineering, Southwest Jiaotong University, Chengdu 610031, \\ PR China \\ ${ }^{b}$ Applied Mechanics and Structure Safety Key Laboratory of Sichuan Province, Southwest \\ Jiaotong University, Chengdu, Sichuan 610031, PR China \\ ${ }^{c}$ Institute for Complex Systems and Mathematical Biology King's College, University of \\ Aberdeen, Aberdeen AB24 3UE, United Kingdom
}

\begin{abstract}
Considering a class of quasiperiodically forced piecewise smooth systems, we uncover a dynamic phenomenon in which strange nonchaotic attractors (SNAs) and quasiperiodic attractors coexist in nonsmooth dynamical system, obtaining the domains of attraction of these coexisting attractors in parameter space in order to analyze the global dynamics. The global dynamics analysis demonstrates that SNAs are the transition from quasiperiodic attractors to chaotic attractors. The routes to SNAs, including torus-doubling route, torus fractalization route or, simply, fractal route, and intermittency route, are also investigated. The characteristics of SNAs are described by dynamical invariants such as the Lyapunov exponent, power spectrum, phase sensitivity and rational approximations.
\end{abstract}

Keywords: Piecewise smooth system; Strange nonchaotic attractors; Coexisting attractors; Global dynamics; Phase sensitivity

\section{Introduction}

SNAs have fractal (strange) geometric structure and do not depend on the initial conditions sensitively (nonchaotic). Since Grebogi et al. [1] uncovered SNA, it has become one of the important topics in nonlinear dynamics. Over the years, many experts have studied strange nonchaotic dynamical properties in different dynamical systems. Sathish et al. [2] showed that logical behaviors persisted in an experimental noisy floor, and logical operation can be observed in the system with two quasiperiodic square-wave forcing. The dynamical phenomenon of the logical operation interval is an SNA. Zhang [3] verified the existence of Wada domain of SNAs in Duffing map with quasiperiodic excitation. The results show that the domain of SNAs is a complete Wada domain in a set of parameters of positive lebesgue measures. Ding et al. [4] showed that SNAs in two-dimensional maps have zero Lyapunov exponent in one direction and negative Lyapunov exponent in the other direction. Zhang et al. [5] studied the dynamics of a class of nonautonomous systems, and strange nonchaotic dynamics were illustrated by several representative systems. Cabanas et al. [6] investigated the current-driven magnetization dynamics in spin torque

\footnotetext{
${ }^{1}$ Correspomding author. E-mail addresses: leyuan2003@sina.com(Yuan Yue)
} 
oscillators, and found that there are strange nonchaotic dynamical phenomena in the system. Paul et al. [7] identified the plausibility of the existence of SNAs in a model of two sinusoidal driven LCR dissipative oscillators sharing a common piecewise nonlinearity, and experimental evidence had been offered by means of a real-time electronic circuit to validate the observed results. The generation mechanism of SNAs is complex. It is useful for understanding the mechanism of SNAs from different bifurcation routes [8]. Yue et al. showed that SNAs are found to exist near the bifurcation points of codimension-2 [9] and codimension-3 [10] bifurcations in periodically forced vibro-impact systems. Wang et al. [11] studied the random dynamical system with periodic excitation and found that small noise is responsible for the robustness of SNAs. Ding et al [12] presented analytical evidence that, in quasiperiodically forced systems, the set in parameter space for which the system exhibits SNAs has Cantor-like structure and is enclosed between two critical curves. One of these curves marks the transition from three-frequency quasiperiodic attractors to SNAs; the other marks the transition from SNAs to chaotic attractors. Heagy [13] et al. found SNAs in a duffing oscillator model with two driven frequencies, showing that SNAs can be generated by the torus-doubling route. In addition, there are Heagy-Hammel route [14], fractal route [15, 16, 17], intermittency route [18, 19, 20], and crisis route $[21,22]$. In 2015, strange nonchaotic stars has also been demonstrated in the RR Lyra Constellation, which further illustrates the existence of strange nonchaotic phenomena in nature [23]. The theoretical studies of SNAs has mainly focused on skew product map. Keller [24] studied a class of monotone incremental quasiperiodically forced maps and proved the existence of SNAs. Alsedà et al. [25] generalized the results of [24] to unimodal quasiperiodically forced maps. Glendinning et al. [26] studied quasiperiodically forced map, proved that SNAs are sensitive to initial phase. Osinga et al. [27] studied the quasiperiodic forcing the dynamics of Arnol'd circle map, the result showed that opening and closing of these pockets of multistability are due to saddle-node and pitchfork bifurcations of invariant curves. They also discussed the bifurcation structure of the Arnol'd tongue and included a study of nonsmooth bifurcations that happen for large nonlinearity in the region with SNAs. In this paper, we study the multistability in a quasiperiodically forced piecewise smooth dynamical system, we uncover abundant coexistence of attractors, and investigated many routes can create SNAs.

Up to now, most of the studies on SNAs has been confined to smooth dynamic systems. The nonsmooth systems have abundant nontypical dynamics [28, 29]. It has been found that SNAs can also be generated in the nonsmooth system in several past works $[30,31$, 32, 33]. Shen et al. [30] investigated the relation between the border-collision bifurcations of tori and the creation of SNAs in the nonsmooth system, and pointed out that the truncation of border-collision torus-doubling bifurcation can lead to different types of SNAs, which enriched the dynamic characteristics of SNA. Li et al. [33] considered a class of single-degree-of-freedom gear dynamical system with quasiperiodic forcing, SNAs are shown to exist in the nonsmooth system by numerical methods. Shen et al. [34] verified SNAs in a simple quasiperiodically forced piecewise smooth system with Farey tree, the results show that the phenomenon of jumping discontinuities appear on the smooth torus can create SNAs. In this work, we consider a class of quasiperiodically forced piecewise smooth systems. Firstly, we analyze the global dynamics in the parameter plane, find that SNAs exist in the transition region from quasiperiodic to chaotic. Secondly, the generation mechanism of SNAs is identified, and the strange property of SNAs is analyzed by phase sensitivity and rational approximations. The results show that there are three routes to 
SNAs in this system: torus-doubling route, fractal route and intermittency route. Finally, we uncover a dynamical phenomenon, multistability [27, 35] involving an SNA in which quasiperiodic attractors and SNAs coexist in certain parameter intervals, and obtain the basin of attraction of these coexisting attractors.

\section{The quasiperiodically forced piecewise smooth system}

We consider the following piecewise smooth map [36],

$$
f: x \rightarrow f(x)=\left\{\begin{array}{ll}
f_{1}(x)=r x, & \text { if } 0 \leq x<\bar{x} ; \\
f_{2}(x)=a x(1-x), & \text { if } \bar{x} \leq x \leq 1 ;
\end{array} \quad \bar{x}=1-\frac{r}{a},\right.
$$

where $a>3,1<r<3$. In this paper, we consider the piecewise smooth system (1) with quasiperiodic excitation, given as follows,

$$
\begin{cases}x_{n+1}=\left(r+\varepsilon \cos 2 \pi \phi_{n}\right) x_{n}, & \text { if } 0 \leq x_{n}<\bar{x} \\ x_{n+1}=\left(a+\varepsilon \cos 2 \pi \phi_{n}\right) x_{n}\left(1-x_{n}\right), & \text { if } \bar{x} \leq x_{n} \leq 1 ; \bar{x}=1-\frac{r}{a} \\ \phi_{n+1}=\bmod \left(\phi_{n}+\omega, 1\right), & \end{cases}
$$

where $\omega=\frac{\sqrt{5}-1}{2}$. $a, r$ and $\varepsilon$ are parameters, and $\varepsilon \cos 2 \pi \phi$ is a small perturbation term.

Because the map (2) is a circle translation in the $\phi$ direction, the Lyapunov exponent in the $\phi$ direction is always zero. The Lyapunov exponent in the $x$ direction is calculated as

$$
\lambda_{x}=\lim _{N \rightarrow \infty} \frac{1}{N} \sum_{i=1}^{N} \log \left|\frac{\partial f}{\partial x_{i}}\right| .
$$

We can determine that the attractor is chaotic $\left(\lambda_{x}>0\right)$ or nonchaotic $\left(\lambda_{x}<0\right)$. There are some tangent bifurcation points in SNAs, where the derivative of these bifurcation points with respect to the phase is infinite, showing that the attractor is nonsmooth [37]. From map (2) we get the recurrence relation

$$
\frac{\partial x_{n+1}}{\partial \phi}=\left\{\begin{array}{lc}
-2 \pi \varepsilon x_{n} \sin 2 \pi \phi_{n}+\left(r+\varepsilon \cos 2 \pi \phi_{n}\right) \frac{\partial x_{n}}{\partial \phi}, & \text { if } 0 \leq x_{n}<\bar{x} \\
-2 \pi \varepsilon x_{n}\left(1-x_{n}\right) \sin 2 \pi \phi_{n}+\left(a+\varepsilon \cos 2 \pi \phi_{n}\right)\left(1-2 x_{n}\right) \frac{\partial x_{n}}{\partial \phi} & \text { if } \bar{x} \leq x_{n} \leq 1
\end{array}\right.
$$

So starting from the initial derivative $\frac{\partial x_{n}}{\partial \phi}$, we get derivatives at all points of the trajectory [37]

$$
\frac{\partial x_{N}}{\partial \phi}= \begin{cases}\sum_{k=1}^{N}-2 \pi \varepsilon x_{k-1} \sin 2 \pi \phi_{k-1} R_{N-k}\left(x_{k}, \phi_{k}\right)+R_{N}\left(x_{0}, \phi_{b}\right) \frac{\partial x_{0}}{\partial \phi} & \text { if } 0 \leq x_{n}<\bar{x} \\ \sum_{k=1}^{N}-2 \pi \varepsilon x_{k-1}\left(1-x_{k-1}\right) \sin 2 \pi \phi_{k-1} R_{N-k}\left(x_{k}, \phi_{k}\right)+R_{N}\left(x_{0}, \phi_{0}\right) \frac{\partial x_{0}}{\partial \phi} & \text { if } \bar{x} \leq x_{n} \leq 1\end{cases}
$$


where

$$
R_{M}\left(x_{m}, \phi_{m}\right)= \begin{cases}\prod_{i=0}^{M-1} r+\varepsilon \cos 2 \pi \phi_{m+i}, & \text { if } 0 \leq x_{n}<\bar{x} \\ \prod_{i=0}^{M-1}\left(a+\varepsilon \cos 2 \pi \phi_{m+i}\right)\left(1-2 x_{m+i}\right), & \text { if } \bar{x} \leq x_{n} \leq 1\end{cases}
$$

$N$ being the number of iterations. According to Ref. [37], $R_{N} \approx \pm \exp \left(\lambda_{x} N\right)$. If the attractor is a nonchaotic attractor with $N \rightarrow+\infty$, then $\lambda_{x}<0$, and $R_{N}\left(x_{0}, \phi_{0}\right) \frac{\partial x_{0}}{\partial \phi}$ is a small quantity. Then Eq. (4) can be expressed as

$$
\frac{\partial x_{N}}{\partial \phi} \approx S^{N} \equiv \begin{cases}\sum_{k=1}^{N}-2 \pi \varepsilon x_{k-1} \sin 2 \pi \phi_{k-1} R_{N-k}\left(x_{k}, \phi_{k}\right), & \\ \sum_{k=1}^{N}-2 \pi \varepsilon x_{k-1}\left(1-x_{k-1}\right) \sin 2 \pi \phi_{k-1} R_{N-k} & \left(x_{k}, \phi_{k}\right), \\ & \text { if } \bar{x} \leq x_{n} \leq 1 .\end{cases}
$$

$S^{N}$ tends to infinite with $N \rightarrow+\infty$, and the attractor is nonsmooth, which characterizes that the attractor to be strange.

The maximum value of $S^{N}$ after $N$ iterations is denoted by

$$
\tau^{N}=\max _{0 \leq n \leq N}\left\{S^{N}\right\}
$$

$\tau^{N} \approx N^{\mu}$, where $\mu$ is the phase sensitivity exponent [37]. If the number of iterations increases, the value of $\tau^{N}$ increases accordingly. If $S^{N}$ tends to infinite with $N \rightarrow+\infty$, then the attractor has infinite derivate with respect to the phase. In such cases, the attractor is strange (i.e. nonsmooth).

The power spectrum is also used to identify the dynamics. If the system exhibits periodic or quasiperiodic behavior, the power spectrum is discrete. If the system exhibits chaotic or random behavior, the power spectrum is continuous [10]. However, for the SNAs, a singular continuum spectrum appears between discrete and continuous $[10,38$, 39]. The Fourier transform is defined as follows,

$$
S(\omega, N)=\sum_{n=1}^{N} x_{n} e^{i 2 \pi m \omega},
$$

and the power spectrum of the attractor is defined as

$$
P_{\omega}=\lim _{N \rightarrow \infty}|S(\omega, N) / N|^{2} .
$$

\section{Global dynamics and SNAs}

\subsection{Global dynamical characteristics}

First, $a$ and $\varepsilon$ are taken as control parameters. For $r=1.95$, the global dynamics is shown in Figs. 1(a) and (b). The range of $a$ is $a \in[3,4]$ in Figs. 1(a) and (b), the range of $\varepsilon$ is $\varepsilon \in[0.2,0.6]$ in Fig. 1(a), and the range of $\varepsilon$ is $\varepsilon \in[0,0.2]$ in Fig. 1(b). The quasiperiodic 
area is denoted by white colour. Because the Lyapunov exponent in the $\phi$ direction is always 0 , the criterion for the quasiperiodic attractor is that the Lyapunov exponent $\lambda_{x}$ be negative. The red curve separates one-torus (1T) quasiperiodic attractor area from the two-tori (2T) quasiperiodic attractor area. When the parameters pass through the red curve, torus-doubling of the quasiperiodic attractor takes place. In Fig. 1(b), for the parameter $\varepsilon \in[0,0.02]$, there is a $3 \mathrm{~T}$ quasiperiodic attractor. SNAs exists between quasiperiodic attractors and chaotic attractors, which are shown in light gray colour. The criterion of SNAs is that the Lyapunov exponent $\lambda_{x}$ be less than zero, and for the phase sensitive exponent $\mu$ not to tend to zero [37]. Chaotic attractors have positive Lyapunov exponent in the $x$ direction, which are shown in gray colour. The escape regimes are shown in black colour. Second, $a$ and $r$ are taken as control parameters. For $\varepsilon=0.3$, the global dynamics is shown in Fig. 1(c). There is a discontinuous quasiperiodic attractor region near the blue curve in Fig. 1(c) (see Section 3.3 for a detailed analysis). Because there is only the linear term of $x$ in the region $0 \leq x<\bar{x}$, the dynamical characteristics of linear systems with quasiperiodic driven do not change with the change of parameter $r$. The red curve separates the $1 \mathrm{~T}$ quasiperiodic attractor from the $2 \mathrm{~T}$ quasiperiodic attractor. When the parameters pass through the red curve, torus-doubling of the quasiperiodic attractor takes place.

\subsection{Strange nonchaotic attractors}

Now let $r=1.95$ and $\varepsilon=0.3$. $a$ is to be taken as control parameter. The evolution of attractors can be observed in the $\left(\phi_{n}, x_{n}\right)$ plane as the parameter $a$ is varied, as shown in Fig. 2. The number of iterations is 50,000, discarding the first 20,000 iterations and then plotting the next 30,000 ones. For $a=3.1$, the attractor is $1 \mathrm{~T}$ quasiperiodic attractor, as shown in Fig. 2(a). For $a=3.15$, the attractor is $2 \mathrm{~T}$ quasiperiodic attractor, as shown in Fig. 2(b). For $a=3.26$, the $2 \mathrm{~T}$ quasiperiodic attractor begins to wrinkle, and some regions become nonsmooth, as shown in Fig. 2(c). The appearance of wrinkling is a fractalization process, a precursor towards the SNA. For $a=3.27$, the attractor loses smoothness completely, and owns the fractal property, as shown in Fig. 2(d). Here SNA is generated by the fractal route (see Section 4.2 for detailed analysis), and the Lyapunov exponent $\lambda_{x}=-0.0098<0$. As shown in Fig. 3(a), for $a=3.27$, the Lyapunov exponent fluctuates about zero for some time but eventually converges to a negative value, which guarantees the strange and nonchaotic properties of SNAs in Fig. 2(d). In addition, the singular continuous power spectrum shows that the attractor is an SNA, as shown in Fig. 4(a). For $a=3.31$, the Lyapunov exponent $\lambda_{x}$ converges to 0.0432 (Fig. 3(b)) and the power spectrum is continuous (Fig. 4(b)), indicating that the attractor shown in Fig. $2(\mathrm{e})$ is chaotic.

\subsection{The discontinuous quasiperiodic phenomena}

There are not only continuous quasiperiodic orbits but also discontinuous quasiperiodic orbits in nonsmooth systems. For example, for $r \in[1.6,1.88]$ in Fig. 1(c), there are discontinuous quasiperiodic orbits, and then SNAs occur with the change of parameter $a$ near the blue curve. Let $a=3.2$ and $\varepsilon=0.3$, taking $r$ as the control parameter. For $r=1.7$, the attractor is a discontinuous $2 \mathrm{~T}$ quasiperiodic, as shown in Fig. 5(a). The discontinuous $2 \mathrm{~T}$ quasiperiodic attractor becomes SNAs as shown in Fig. 5(b) when $r=$ 1.62. The results show that the discontinuous quasiperiodic attractor exists in nonsmooth systems, which can turn into SNAs with the change of parameter. 


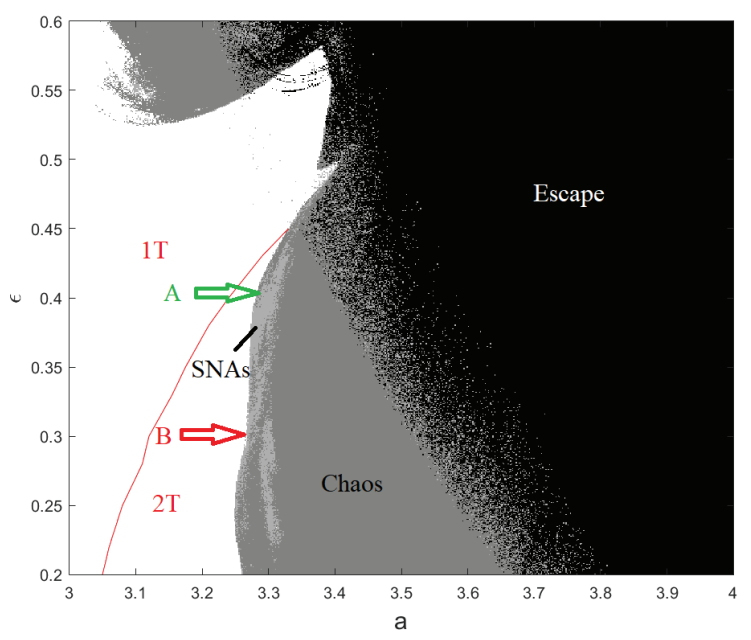

(a)

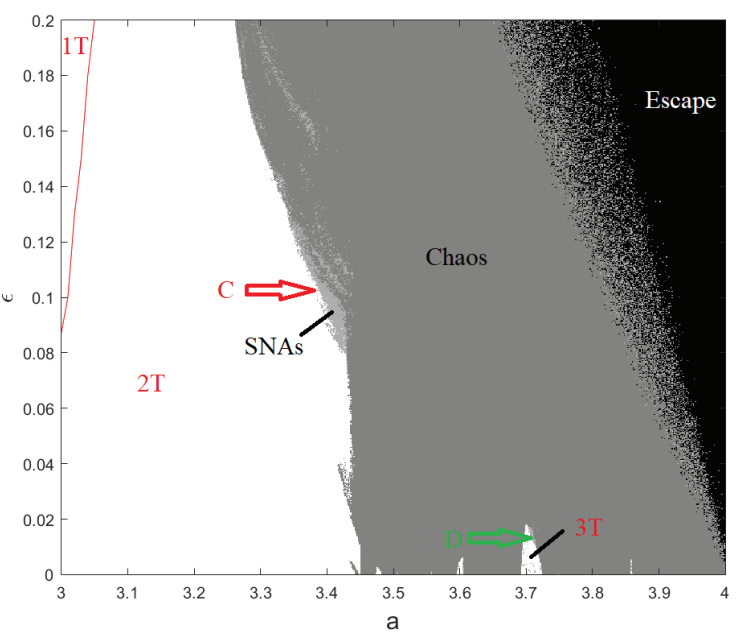

(b)

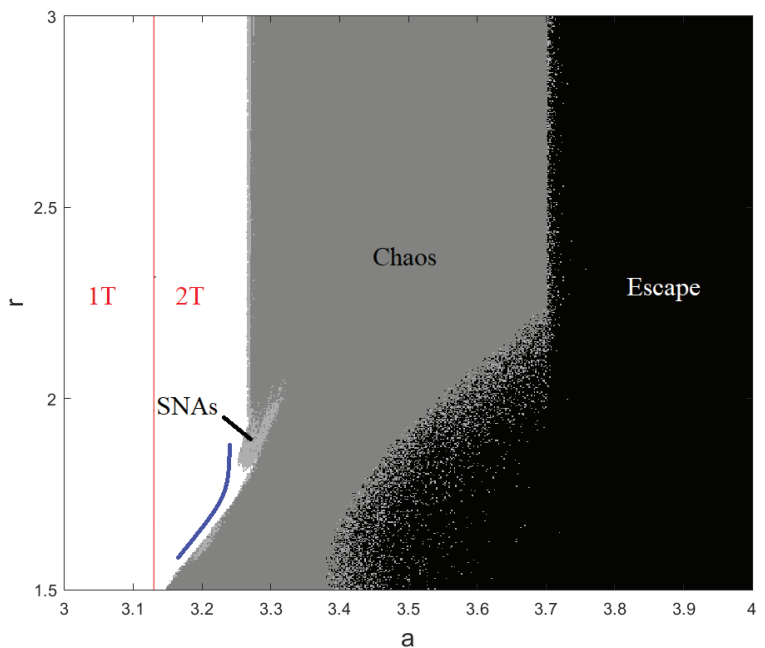

(c)

Figure 1: (a) The phase diagram in the plane $(a, \varepsilon), r=1.95, a \in[3,4], \varepsilon \in[0.2,0.6]$; (b) the phase diagram in the plane of $(a, \varepsilon), r=1.95, a \in[3,4], \varepsilon \in[0,0.2]$; (c) the phase diagram in the plane of $(a, r)$, $\varepsilon=0.3, a \in[34], r \in[1.5,3]$. The red and green arrows indicate strange nonchaotic attractors. 


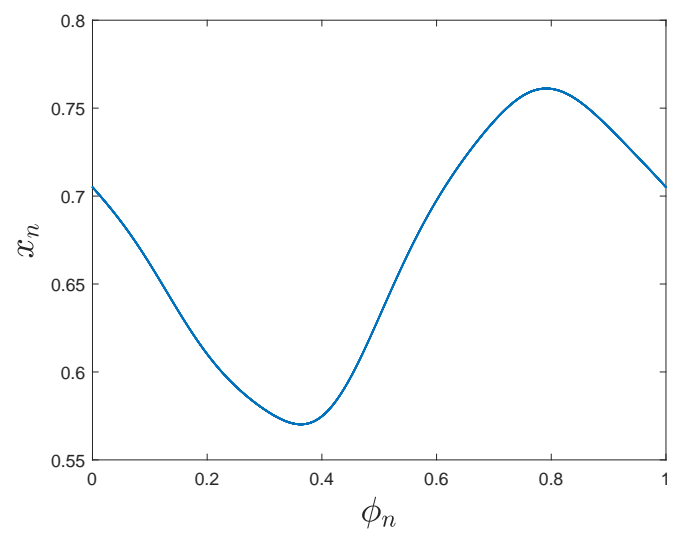

(a) $a=3.1$

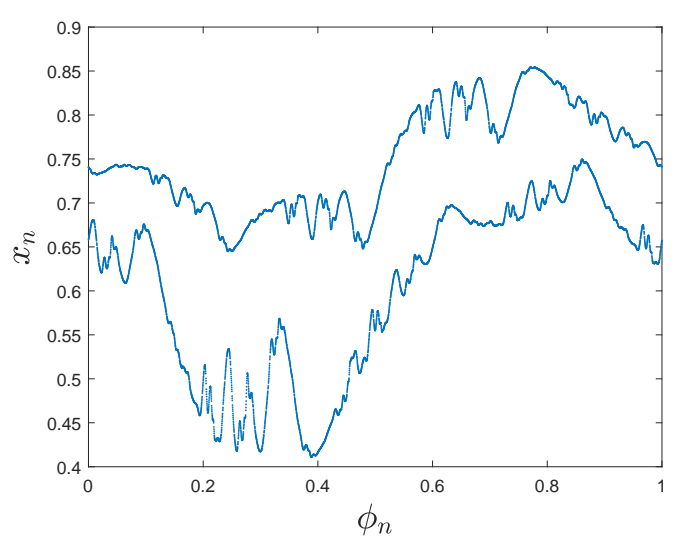

(c) $a=3.26$

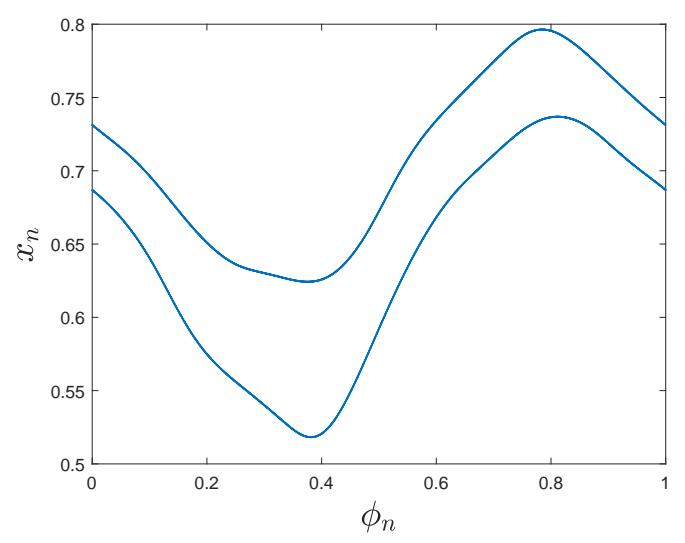

(b) $a=3.15$

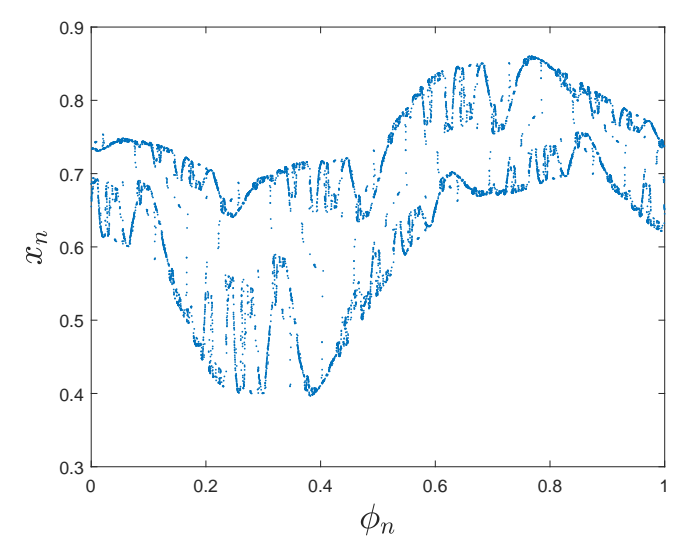

(d) $a=3.27$

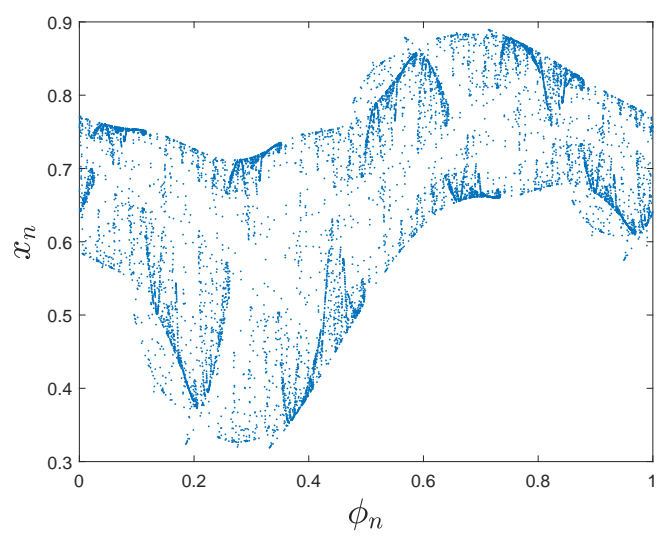

(e) $a=3.31$

Figure 2: For $r=1.95, \varepsilon=0.3$, the phase diagram in the $\left(\phi_{n}, x_{n}\right)$ plane.

\subsection{Determining the strange property of attractors}

\subsubsection{Phase sensitivity property}

The strange property can be characterized by the phase sensitivity property effectively [37]. Here we take SNAs in Fig. 2 as an example. $\tau^{N}$ is the maximum derivative of $x$ with respect to the phase $\phi$ as in Eq. (10). We get $\tau^{N} \approx N^{\mu}$ [37], and $\mu$ is the phase sensitivity exponent. For $a=3.15$, the attractor is $2 \mathrm{~T}$ quasiperiodic attractor. For $a=3.27$, the 


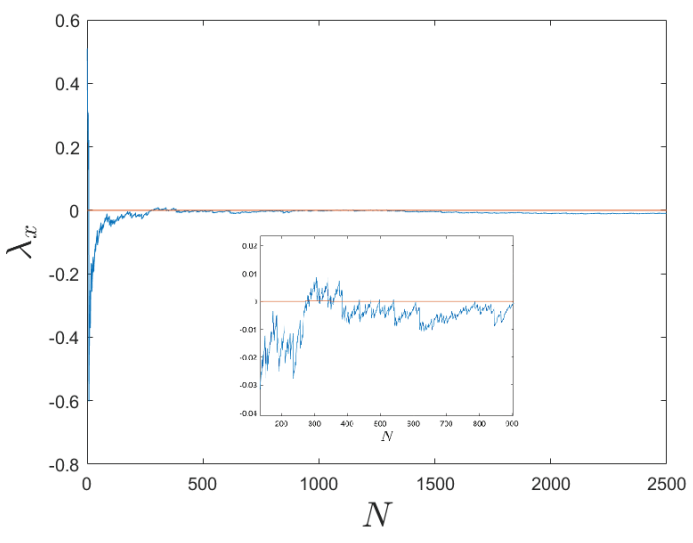

(a) $a=3.27$

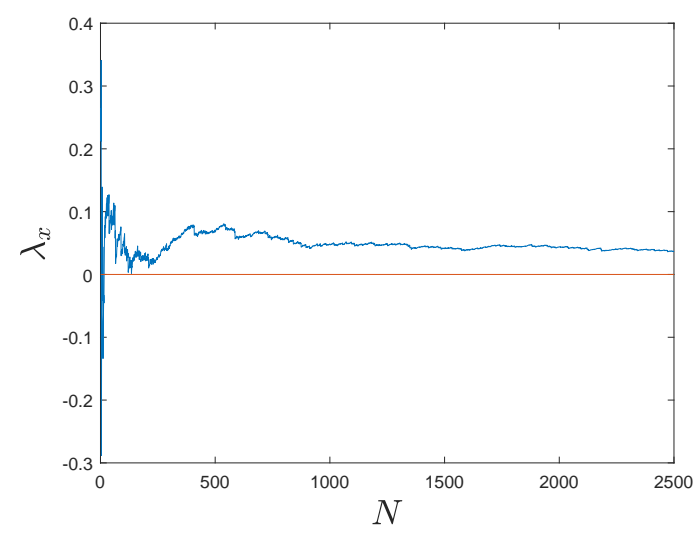

(b) $a=3.31$

Figure 3: For $r=1.95, \varepsilon=0.3$, the Lyapunov exponent in the $x$ direction.

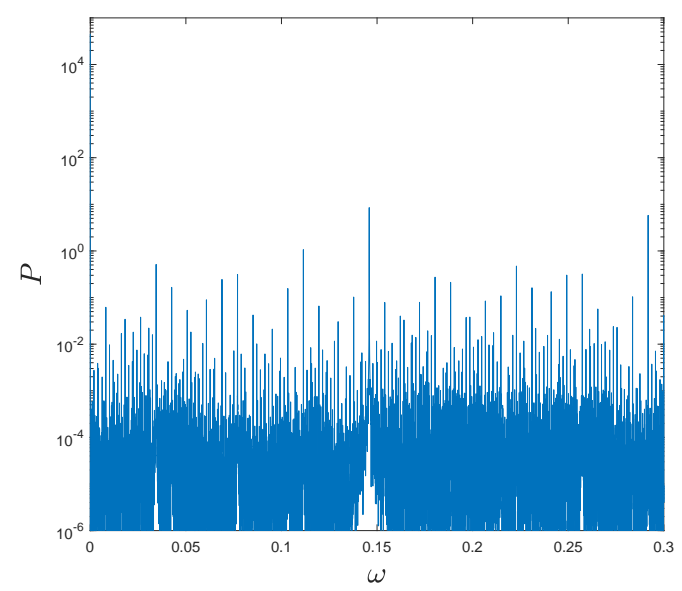

(a) $a=3.27$

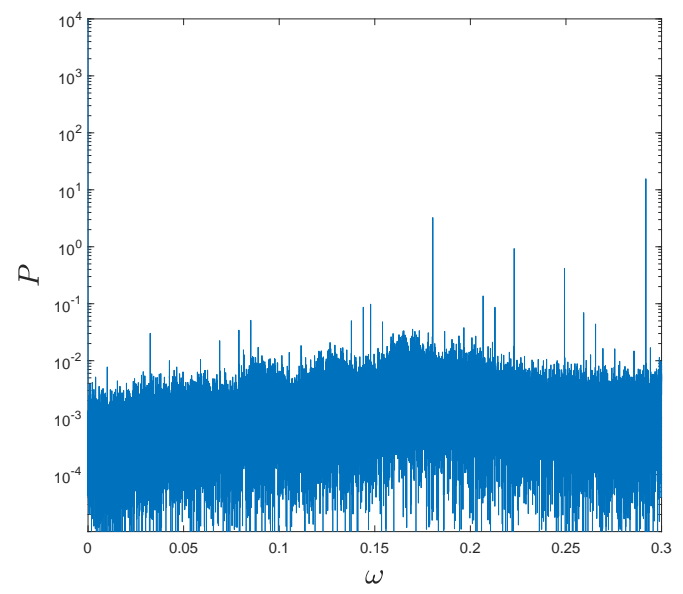

(b) $a=3.31$

Figure 4: For $r=1.95, \varepsilon=0.3$, the power spectrum.

attractor is an SNA and nonsmooth, the derivative of $x$ with respect to the phase $\phi$ tends to infinite. In the numerical calculation, 10,000 iterations and 100,000 iterations are selected to obtain the change image of $\tau^{N}$ with the number of iterations. As the number of iterations increases, $\tau^{N}$ increases continuously, as shown in Figs. 6(a) and (b). $\tau^{N}$ tends to infinite with $N \rightarrow \infty$, and the phase sensitive exponents are $\mu=1.0748$ and $\mu=1.4233$, respectively. If the attractor is quasiperiodic $(a=3.15), \tau^{N}$ has a bounded value, and the phase sensitive exponent $\mu$ tends to zero in Figs. 6(a) and (b). For SNAs $(a=3.27)$, the attractors are nonsmooth, $\tau^{N}$ tends to infinite. This property can be used to distinguish SNAs from quasiperiodic attractors. If the attractor does not have a finite derivative with respect to the phase, the attractor is nonsmooth (i.e. strange).

\subsubsection{Rational approximations}

The method of approximating irrational frequency with rational frequency can also be used to distinguish SNAs [37]. For the golden mean value of $\omega$, the ratios of Fibonacci numbers $\left(\omega_{k}=F_{k+1} / F_{k}, F_{k+1}=F_{k}+F_{k-1}, F_{1}=1, F_{2}=1\right)$ are approximations. When 


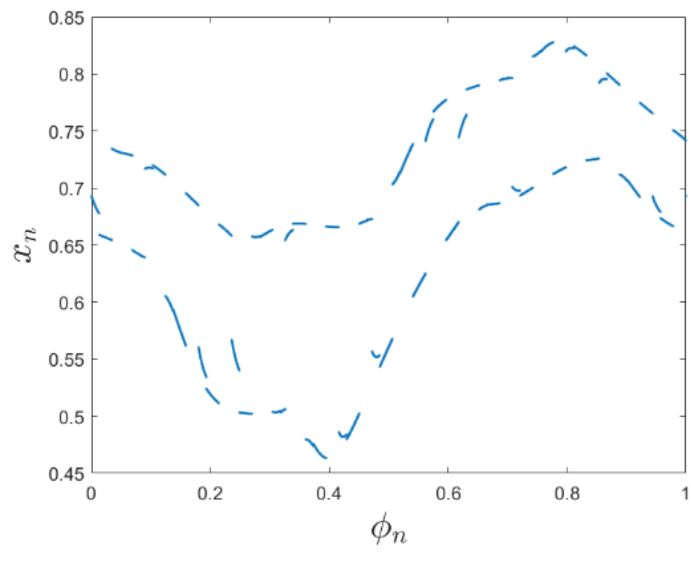

(a) $r=1.7$

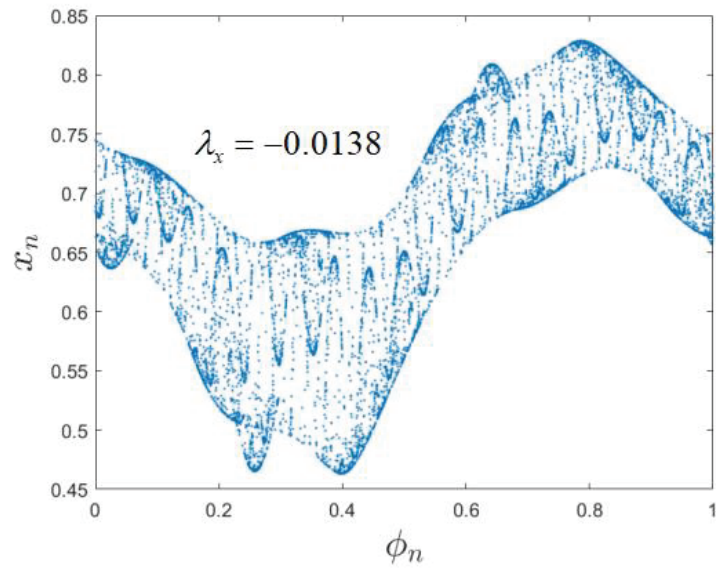

(b) $r=1.62$

Figure 5: The parameters: $a=3.2, \varepsilon=0.3$, the phase diagram in the $\left(\phi_{n}, x_{n}\right)$ plane. (a) Discontinuous qusaiperiodic attractor, (b) SNAs.

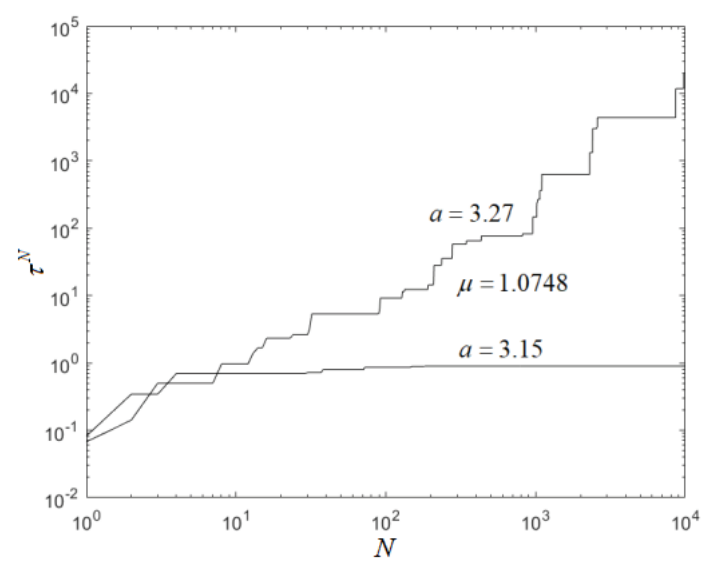

(a) 10000 iterations

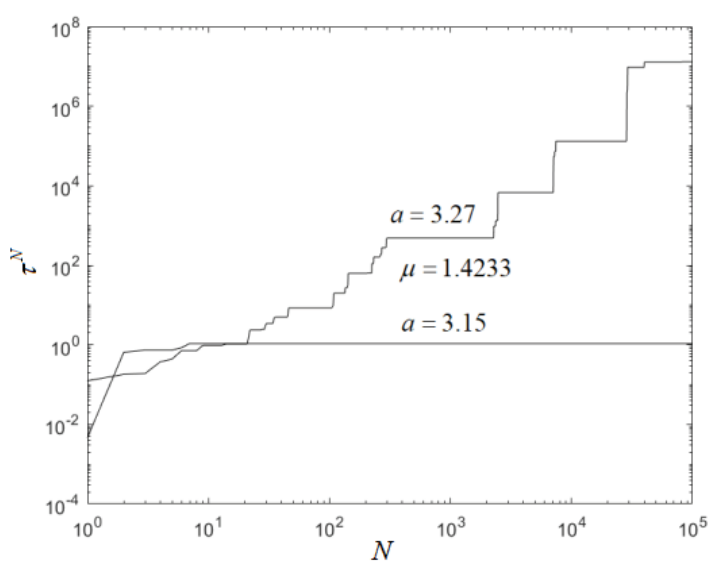

(b) 100000 iterations

Figure 6: Phase sensitivity functions: $r=1.95, \varepsilon=0.3$.

the different rational approximations are 34/55, 610/98, 987/1597, 4181/6765, the phase diagrams in the $\left(\phi_{n}, x_{n}\right)$ plane are shown in Figs. 7(a), (b), (c) and (d), respectively. As $\omega_{10}=34 / 55$ and $\omega_{16}=610 / 987$, the order of the attractor approximation is low, which can not present the shape of SNAs, as shown in Figs. $7(\mathrm{a})$ and (b). When $\omega_{17}=987 / 1597$ and $\omega_{19}=4181 / 6765$, the attractors in Figs. $7(\mathrm{c})$ and (d) approximate SNAs in Fig. 2(d). As the order of approximation increases, the number of periodic points also increases, tending to the geometric fractal structure of SNA, and it can approximate the strange nonchaotic property of the quasiperiodic forced system with $k \rightarrow \infty$. As $\omega_{k} \rightarrow(\sqrt{5}-1) / 2$, the number of periodic points tends to infinite, and the structure of the attractor is not a finite set of points and is not piecewise differentiable, which depicts the strangeness of the attractor. 


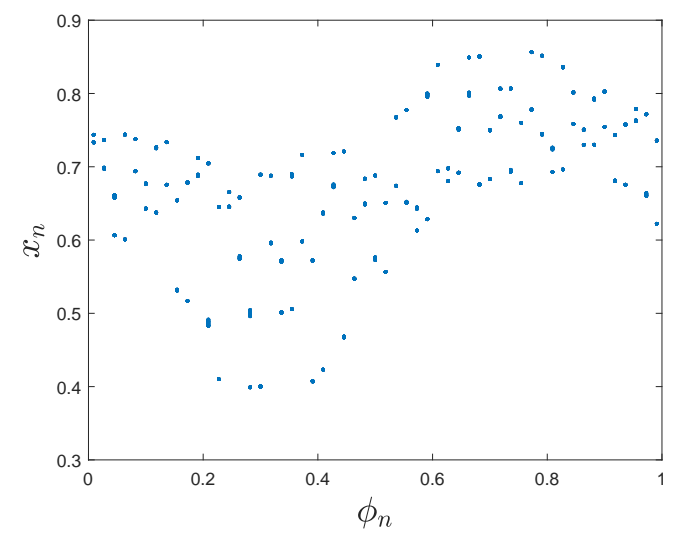

(a) $\omega_{10}=34 / 55$

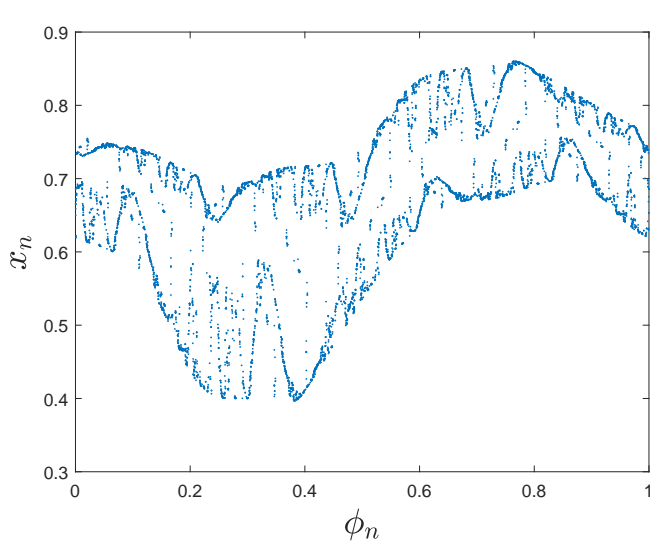

(c) $\omega_{17}=987 / 1597$

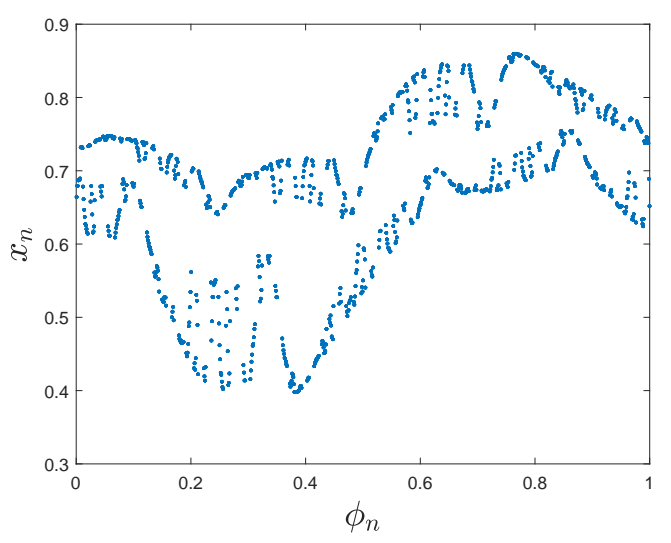

(b) $\omega_{16}=610 / 987$

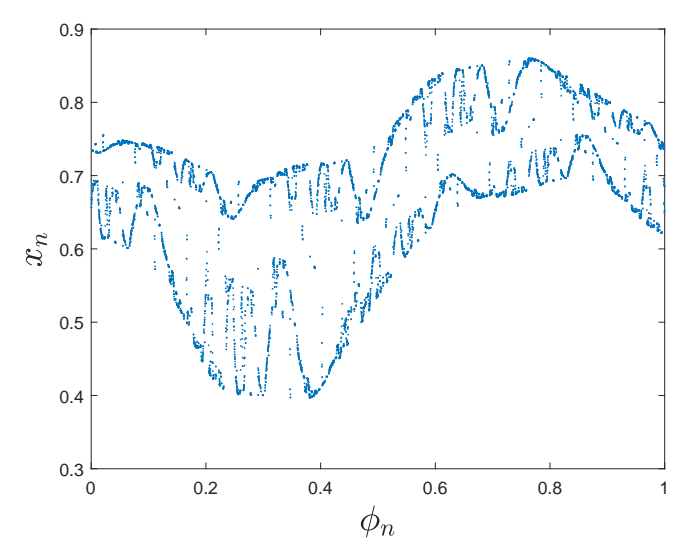

(d) $\omega_{19}=4181 / 6765$

Figure 7: Rational approximations: $r=1.95, \varepsilon=0.3, a=3.27$.

\section{Routes to SNAs}

\subsection{Torus-doubling route}

Let $r=1.9$ and $\varepsilon=0.0001$, taking $a$ as the control parameter. The quasiperiodic attractor of the system generates SNA by torus-doubling in the phase diagram in the $\left(\phi_{n}, x_{n}\right)$ plane, and the evolution process is as follows: 2T(Fig. $\left.8(\mathrm{a})\right) \rightarrow 2 \mathrm{~T}+4 \mathrm{~T}$ (Fig. $8(\mathrm{~b})) \rightarrow 2 \mathrm{~T}+8 \mathrm{~T}$ (Fig. 8(c)) $\rightarrow 2 \mathrm{~T}+16 \mathrm{~T}$ (Fig. 8(d)) $\rightarrow 2 \mathrm{~T}+\mathrm{SNA}($ Fig. 8(e)). As the parameter $a$ is varied, the Lyapunov exponent in the $x$ direction is shown in Fig. 9. For $\lambda_{x}=0$, there are flip bifurcation points in the system, such as A $(a=3.4137)$ and B $(a=3.4206)$ points. When the parameter $a$ passes through the flip bifurcation points, the system goes through a torus-doubling. For $a=3.35$, the attractor is a $2 \mathrm{~T}$ quasiperiodic attractor, as shown in Fig. 8(a). When $a$ is increased to 3.41, there is a coexistence of a $2 \mathrm{~T}$ quasiperiodic attractor (red) and a $4 \mathrm{~T}$ quasiperiodic attractor (blue), as shown in Fig. $8(\mathrm{~b})$. For $a=3.42$, the parameter passes though the flip bifurcation point A, and the system undergoes torus-doubling, $4 \mathrm{~T}$ quasiperiodic attractor becomes $8 \mathrm{~T}$ quasiperiodic attractor. The $2 \mathrm{~T}$ quasiperiodic attractor (red) coexists with $8 \mathrm{~T}$ quasiperiodic attractor (blue), as shown in Fig. 8(c). For $a=3.422$, the parameter passes though the flip bifurcation point $\mathrm{B}$, and the system undergoes torus-doubling. 8T quasiperiodic attractor becomes $16 \mathrm{~T}$ quasiperiodic attractor. The $2 \mathrm{~T}$ quasiperiodic attractor (red) coexists with 
16T quasiperiodic attractor (blue), as shown in Fig. 8(d). As $a$ is increased to 3.42265, 16T quasiperiodic attractor becomes SNA, as shown in Fig. 8(e). Here the Lyapunov exponent $\lambda_{x}=-0.003, \tau^{N}$ tends to infinite, and the phase sensitivity exponent $\mu=12.725$, as shown in Fig. 8(f), which indicate the strange and nonchaotic property of the attractor. Then SNAs can be generated by torus-doubling route in piecewise smooth systems.

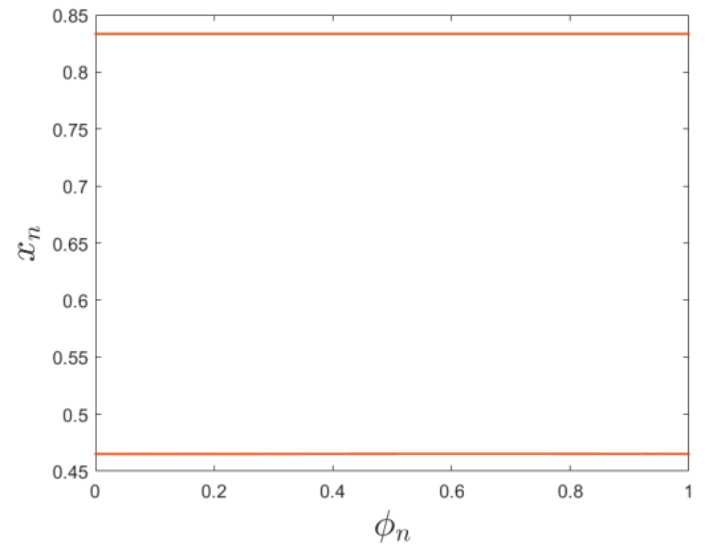

(a) $a=3.35$

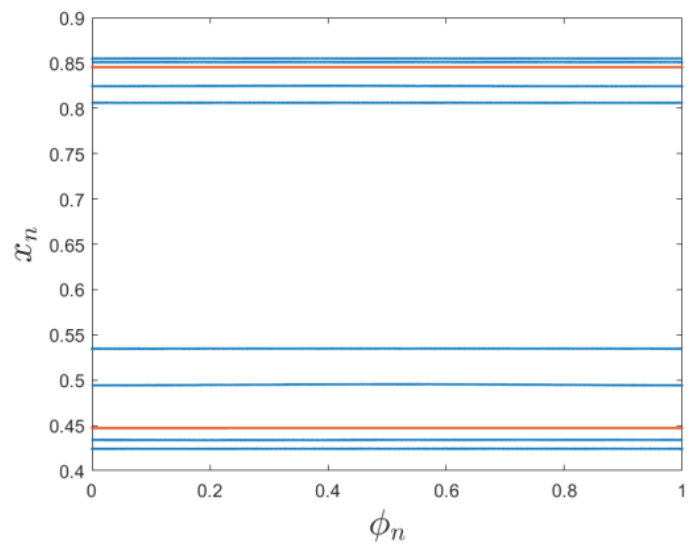

(c) $a=3.42$

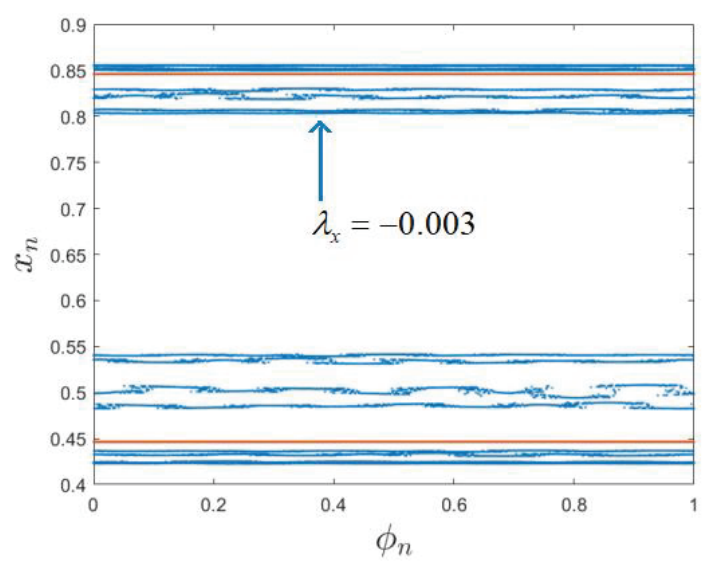

(e) $a=3.42265$

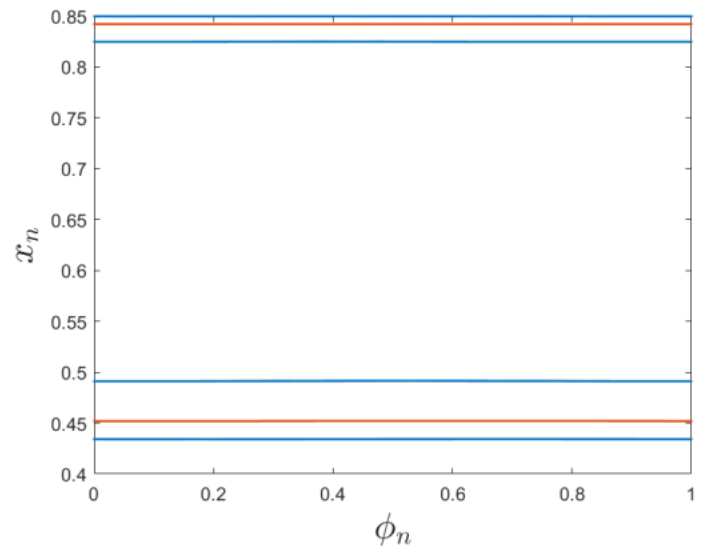

(b) $a=3.41$

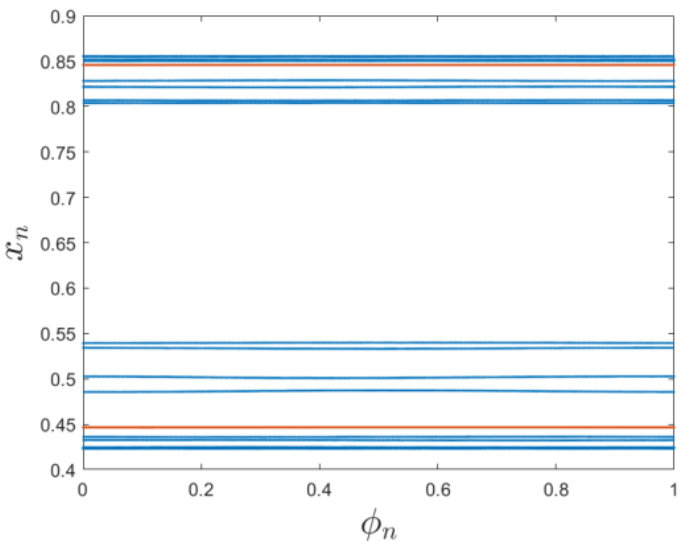

(d) $a=3.422$

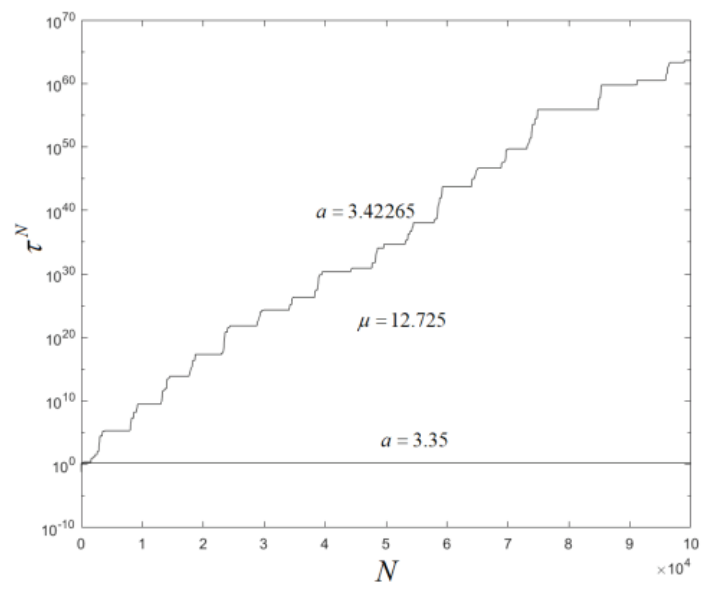

(f) Phase sensitivity functions

Figure 8: Torus-doubling route to SNA: $r=1.9, \varepsilon=0.0001$, the phase diagrams in $\left(\phi_{n}, x_{n}\right)$. 


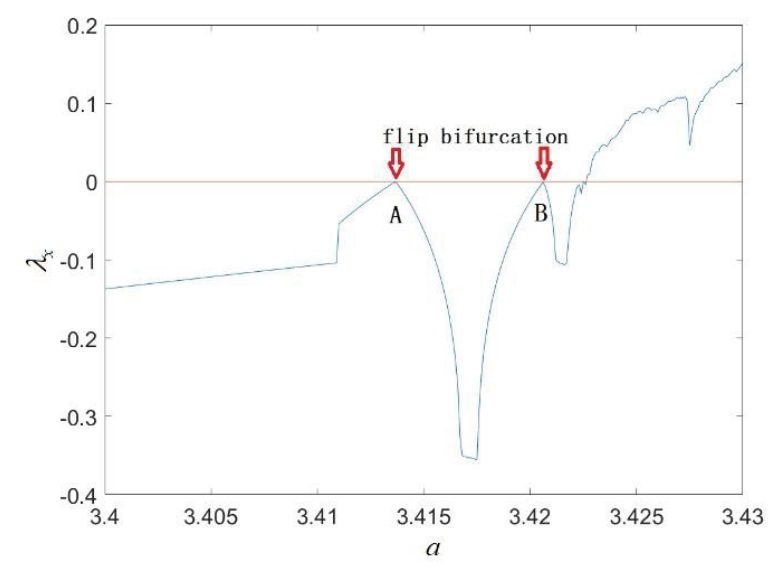

Figure 9: For $r=1.9, \varepsilon=0.0001$, the Lyapunov exponent $\lambda_{x}$ with the change of $a$.

\subsection{Fractal route}

Fractal route to SNAs is common in smooth systems. With the change of parameters, the torus begins to wrinkle, gradually losing its smoothness, and eventually evolving into SNAs. Here the piecewise smooth system can also generate SNAs by the fractal route. In Fig. 1(a), two directions are selected to verify it in the parameter space, one is $\mathrm{A}(r=1.95$, $\varepsilon=0.4)$ and the other is $\mathrm{B}(r=2.5, \varepsilon=0.3)$. For direction $\mathrm{A}$, the phase diagrams are shown in Figs. 10(a) ( $a=3.277: 2 \mathrm{~T}$ quasiperiodic attractor) and (b) ( $a=3.285$ : SNA). For direction B, the phase diagrams are shown in Figs. 10(c) $(a=3.265$ : 2T quasiperiodic attractor) and (d) ( $a=3.27$ : SNA). For the two cases, $2 \mathrm{~T}$ quasiperiodic attractors in Figs. 10 (a) and (c) do not double, but wrinkle gradually with the change of parameter, showing a fractal shape, and evolving into SNAs in Figs. 10(b) and (d), respectively. The strange property of SNA can be characterized by the phase sensitivity property and rational approximations. Phase sensitivity exponents of Figs. 10(b) and (d) are $\mu=2.2813$ and $\mu=0.9326$, as shown in Figs. 11(a) and (b), respectively. At the same time, for the order of the attractor approximation $\omega_{19}=4181 / 6765$, the phase diagrams are shown in Figs. 11(c) and (d), respectively, which tend to the geometric fractal structure. If the order of the attractor approximation is infinite, the strange property of SNA is verified by the infinite approximation. The nonchaotic property is verified by the Lyapunov exponent $\lambda_{x}$. For the system parameters $a=3.285, \varepsilon=0.4$ and $r=1.95$, the Lyapunov exponent $\lambda_{x}$ is -0.0032 . For the system parameters $a=3.27, \varepsilon=0.3$, and $r=2.5$, the Lyapunov exponent $\lambda_{x}$ is -0.0164 . In conclusion, the quasiperiodically forced piecewise smooth systems can also generate SNAs by the fractal route.

\subsection{Type-I intermittency route}

Type-I intermittency route is created by the saddle-node bifurcation. In Fig. 1(b), for $\varepsilon \in\left[\begin{array}{ll}0.011 & 0.016\end{array} \cup[0.0770 .124]\right.$, the quasiperiodic attractor turns into SNA by typeI intermittency route. Two directions are selected to verify in the parameter space, one is $\mathrm{C}(r=1.95, \varepsilon=0.1)$ and the other is $\mathrm{D}(r=1.95, \varepsilon=0.017)$ in Fig. 1(b). In the direction $\mathrm{C}$, for $a=3.34$, the attractor is $2 \mathrm{~T}$ quasiperiodic, as shown in Fig. 12 (a). When $a$ is increased to 3.414 , the approximate shape of the quasiperiodic orbit of the system is still present, but there are many disordered points near the orbit, which are a characteristics of intermittency, as shown in Fig. 12(b). Here the attractor is 


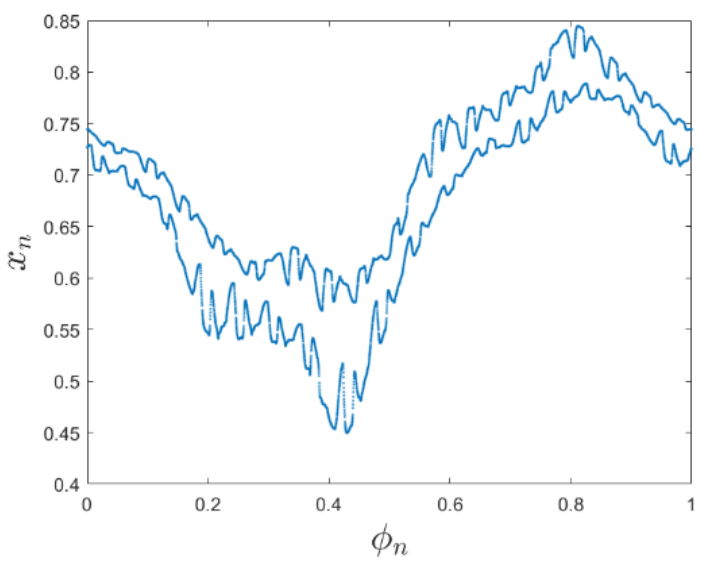

(a) $a=3.277, \varepsilon=0.4, r=1.95$

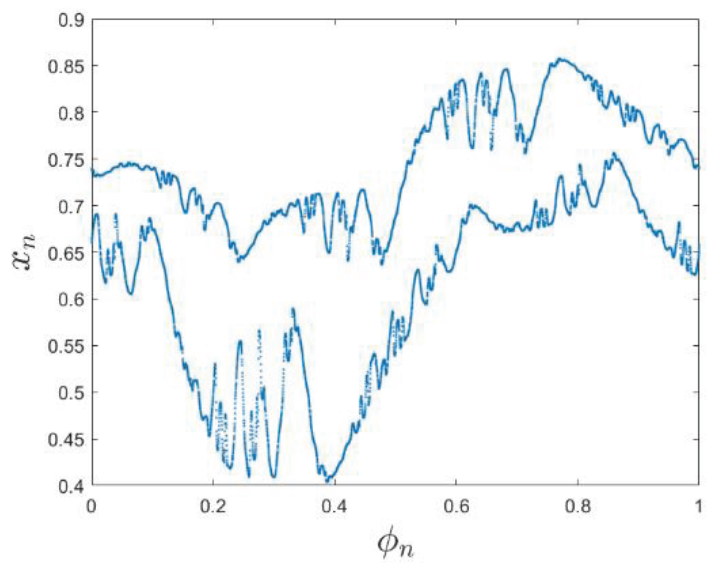

(c) $a=3.265, \varepsilon=0.3, r=2.5$

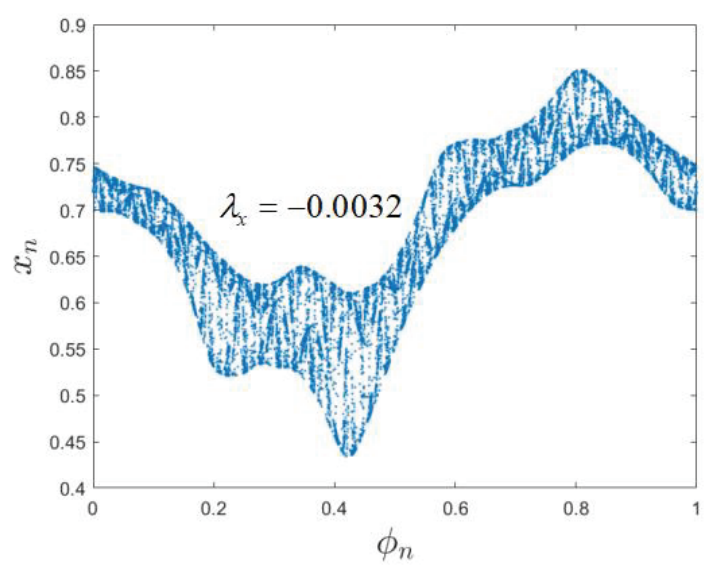

(b) $a=3.285, \varepsilon=0.4, r=1.95$

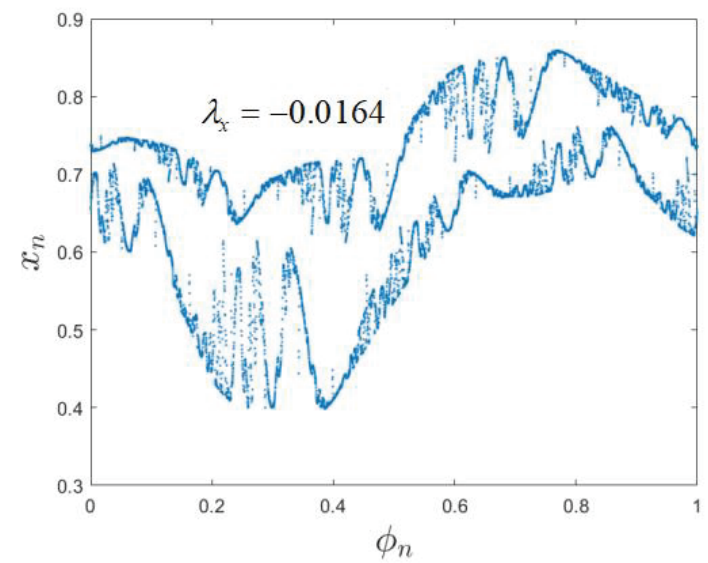

(d) $a=3.27, \varepsilon=0.3, r=2.5$

Figure 10: The fractal route to SNA.

strange nonchaotic in Fig. 12(b). The nonchaotic property is denoted by the Lyapunov exponent $\lambda_{x}=-0.0012$. The strange property of an SNA is characterized by the phase sensitivity and rational approximations. The maximum value of the phase derivative $\tau^{N}$ tends to infinity, and the phase sensitivity exponent $\mu=3.4886$, as shown in Fig. 13(a). For rational approximations, taking the order of the attractor approximation to be $\omega_{19}=4181 / 6765$, the geometric fractal structure of Fig. 13(c) approximates Fig. 12(b). In the direction $\mathrm{D}$, for $a=3.7$, the attractor is a $3 \mathrm{~T}$ quasiperiodic attractor, as shown in Fig. 12(c). As $a$ is increased to 3.7028, the attractor becomes SNA as shown in Fig. 12(d), with the Lyapunov exponent $\lambda_{x}=-0.0238$. The phase sensitivity exponent $\mu=6.3452$ is shown in Fig. 13(b), and the geometric fractal structure of Fig. 13(d) approximates Fig. 12(d). In both cases, SNAs generated by type-I intermittency are derived from a saddle-node bifurcation in the system. In conclusion, SNAs can also be generated by type-I intermittency route in piecewise smooth systems.

\section{Multistability involving quasiperiodic attractors and SNAs}

A few studies on the coexistence of SNAs and quasiperiodic attractors in smooth systems have been reported [2, 40,41]. In this section, the coexistence of SNAs and 


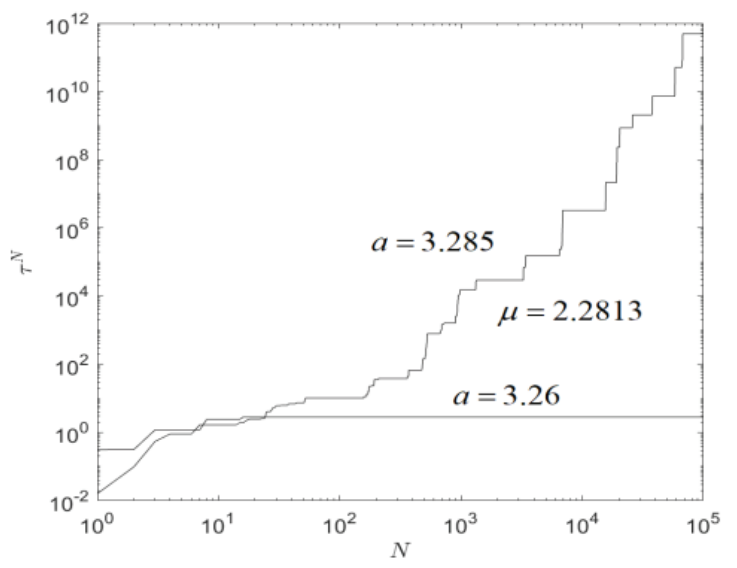

(a)

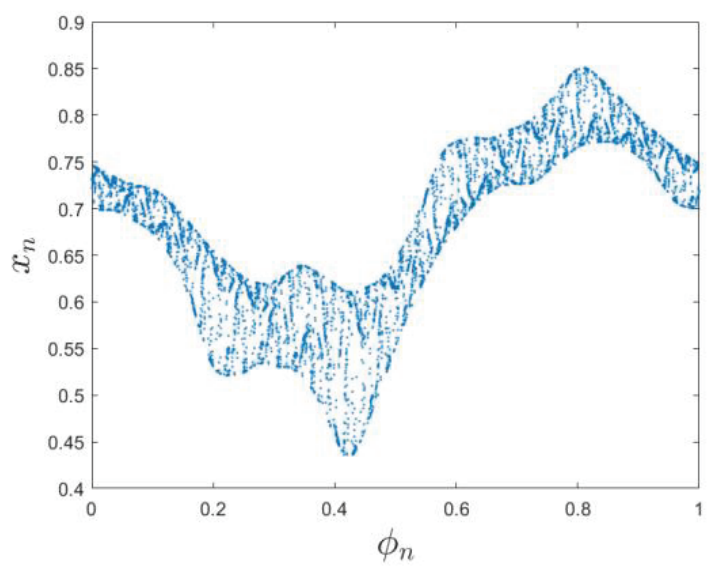

(c) $\omega_{19}=4181 / 6765$

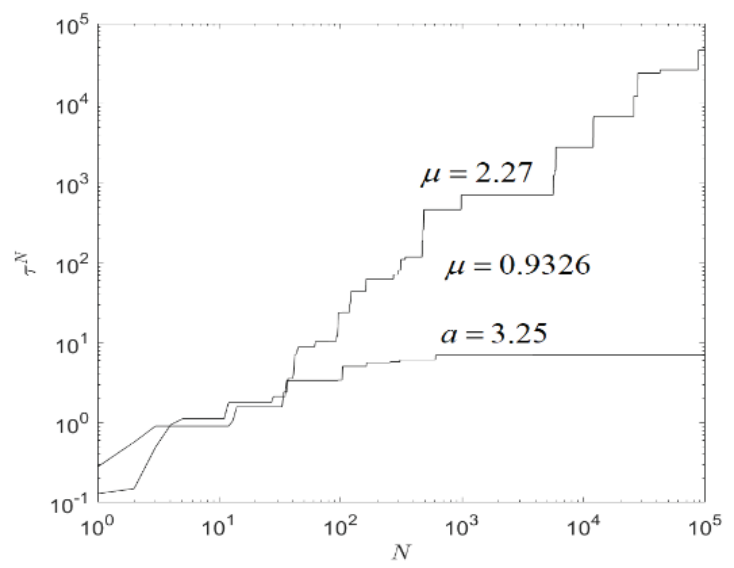

(b)

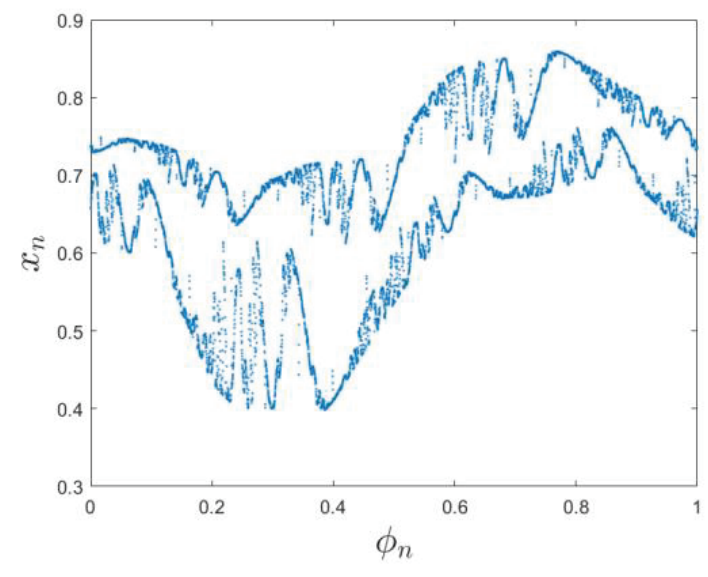

(d) $\omega_{19}=4181 / 6765$

Figure 11: Phase sensitivity functions. (a) $a=3.285, \varepsilon=0.4, r=1.95$, (b) $a=3.27, \varepsilon=0.3, r=2.5$. Rational approximations. (c) $a=3.285, \varepsilon=0.4, r=1.95$, (d) $a=3.27, \varepsilon=0.3, r=2.5$.

quasiperiodic attractors in nonsmooth systems is uncovered. We consider the system parameters (1) $\varepsilon=0.006, r=1.9$, and the parameter $a$ as the control parameter. For $a \in$ [3 3.8 , two different bifurcation diagrams are obtained by taking initial values $\left(x_{0}, y_{0}\right)=$ $(0.1,0.3)$ and $\left(x_{0}, y_{0}\right)=(0.42,0.3)$, as shown in Figs. $14(\mathrm{a})$ and $(\mathrm{b})$, respectively. For $a \in[3.4063 .42]$, Figs. 14(c) and (d) are local enlargements of bifurcation diagrams of Figs. 14(a) and (b), respectively. The corresponding Lyapunov exponential diagrams in the $x$ direction with the range of $a$ shown in Figs. 14(e) and (f), respectively. The results show that for $a \in[3.40953 .415]$, there are the coexisting attractors. The initial value of blue attractor is $\left(x_{0}, y_{0}\right)=(0.1,0.3)$, and that of red attractor is $\left(x_{0}, y_{0}\right)=(0.42,0.3)$.

The phase diagrams in the $\left(\phi_{n}, x_{n}\right)$ plane are shown in Fig. 15. For $a=3.4$, there is a $2 \mathrm{~T}$ quasiperiodic orbit (red orbit), as shown in Fig. 15(a). When $a$ is increased to 3.411, the coexistence of a $2 \mathrm{~T}$ quasiperiodic attractor and a $4 \mathrm{~T}$ quasiperiodic attractor appears, as shown in Fig. 15(b). For $a=3.4127$, the $4 \mathrm{~T}$ quasiperiodic attractor evolves into a SNA with 4 bands, the Lyapunov exponent $\lambda_{x}$ is -0.0017 (blue attractor), and there is the coexistence of a $2 \mathrm{~T}$ quasiperiodic attractor and a SNA with 4 bands, as shown in Fig. 15(c). When $a$ is increased to 3.413, the dynamical characteristics of the system change further. There is the coexistence of a $2 \mathrm{~T}$ quasiperiodic attractor and a chaotic 


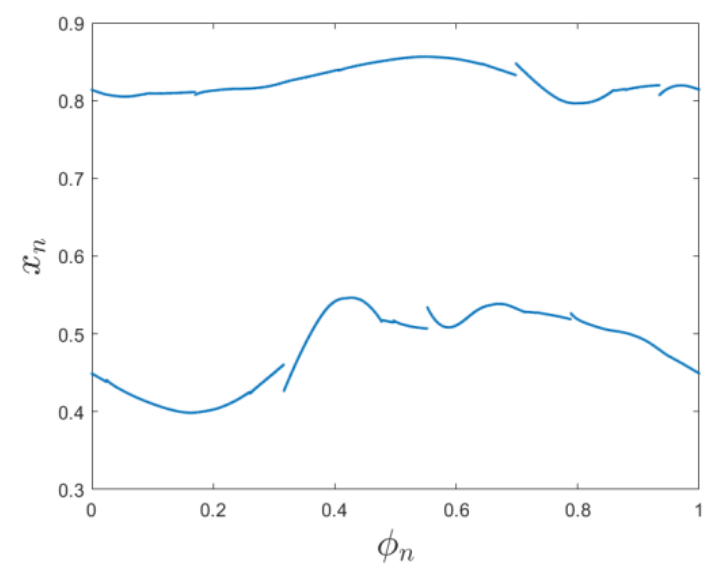

(a) $a=3.34, \varepsilon=0.1, r=1.95$

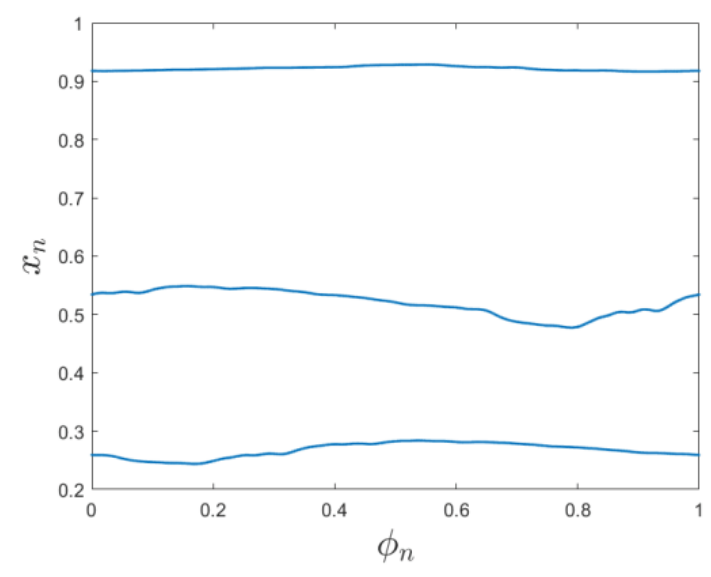

(c) $a=3.7, \varepsilon=0.017, r=1.95$

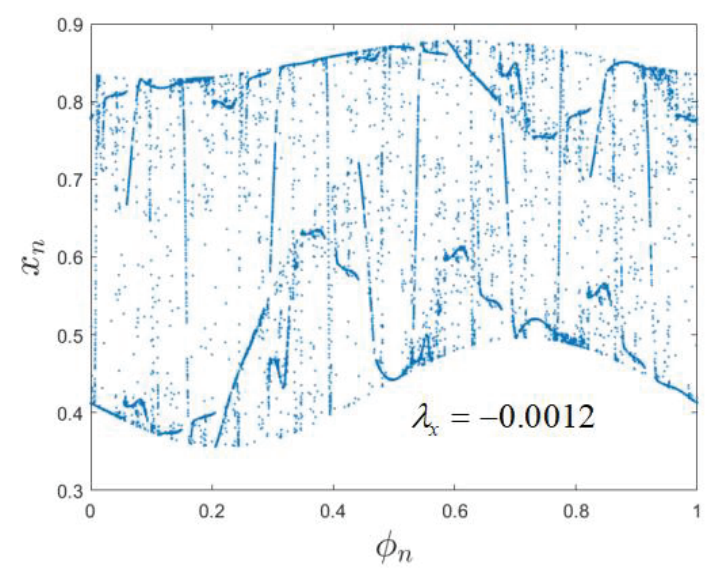

(b) $a=3.414, \varepsilon=0.1, r=1.95$

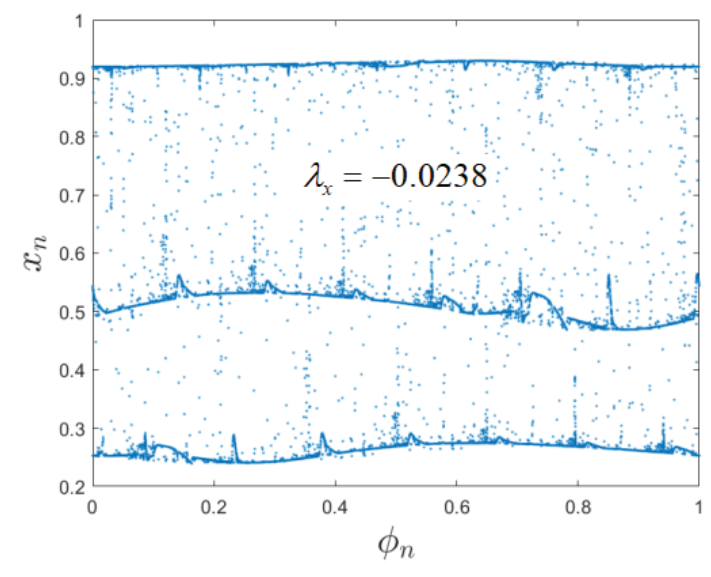

(d) $a=3.7028, \varepsilon=0.017, r=1.95$

Figure 12: Type-I intermittency route to SNA.

attractor with 4 bands, and the Lyapunov exponent of the chaotic attractor with 4 bands is $\lambda_{x}=0.0041$ (blue attractor), as shown in Fig. 15(d). When $a$ is increased to 3.414, the chaotic attractor with 4 bands disappears and only the $2 \mathrm{~T}$ quasiperiodic attractor remains, as shown in Fig. 15(e). As $a$ is increased to 3.41964, the Lyapunov exponent is $\lambda_{x}=-0.034$ (red attractor), and the $2 \mathrm{~T}$ quasiperiodic attractor evolves into a SNA with 2 bands by type-I intermittency route, as shown in Fig. 15(f). For $a=3.41965$, the Lyapunov exponent is $\lambda_{x}=0.0532$, SNA evolves into a chaotic attractor with 2 bands, as shown in Fig. 15(g). For $a=3.4127$, the basin of attraction is shown in Fig. 16. If the initial value is in the yellow region, the orbit will eventually asymptote to the SNA (blue). If the initial value is in the blue region, the orbit finally asymptotes to the $2 \mathrm{~T}$ quasiperiodic attractor (red). When parameter $a$ continues to increase, the crisis phenomena can be observed from the bifurcation diagram, as shown in Fig. 14. For $a \in[3.588,3.596]$, the evolution of the attractor is as follows: chaotic attractor with 4 bands $\rightarrow 5 \mathrm{~T}$ quasiperiodic attractor $\rightarrow$ chaotic attractor with 5 bands. For $a \in[3.682,3.702]$, the evolution of the attractor is as follows: chaotic attractor with 5 bands $\rightarrow$ SNA with 3 bands $\rightarrow 3 \mathrm{~T}$ quasiperiodic attractor $\rightarrow$ SNA with 3 bands $\rightarrow$ chaotic attractor with 3 bands. The generated route of SNA is caused by type-I intermittency.

Now we take the system parameters (2) $\varepsilon=0.0015, r=1.9312$, and the parameter $a$ as 


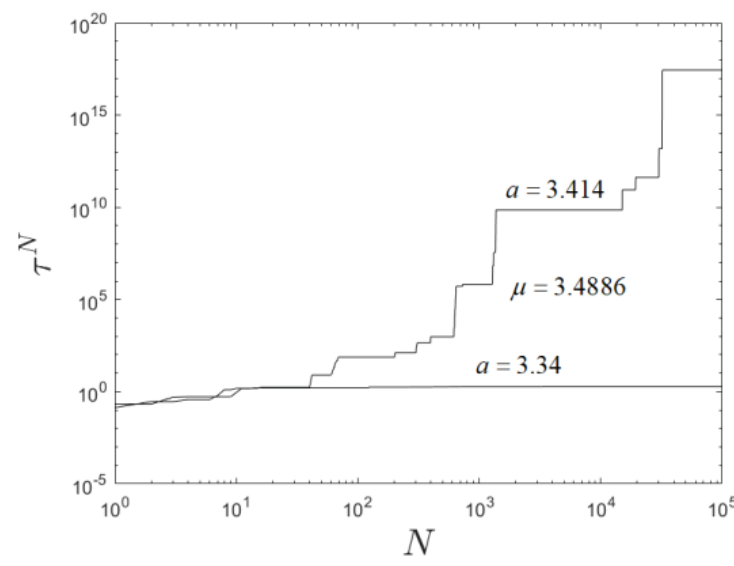

(a)

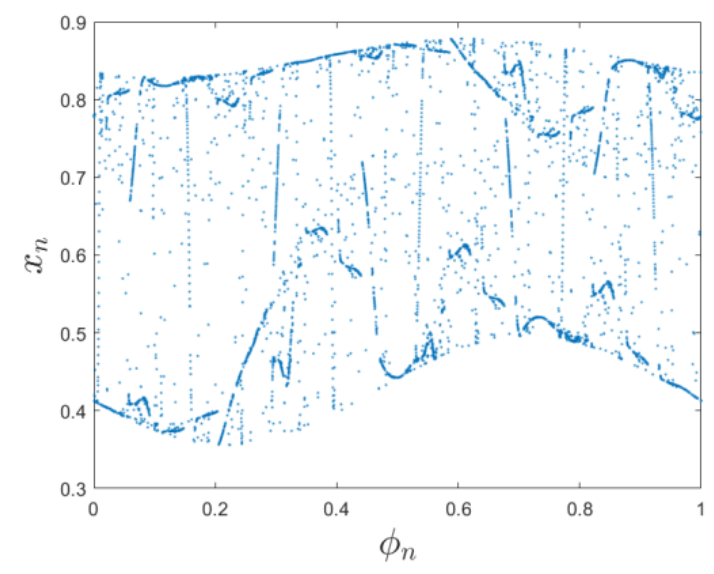

(c) $\omega_{19}=4181 / 6765$

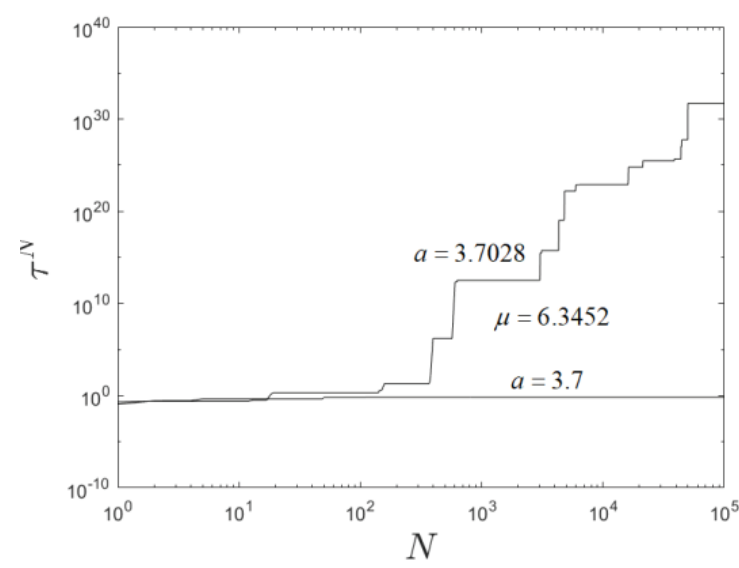

(b)

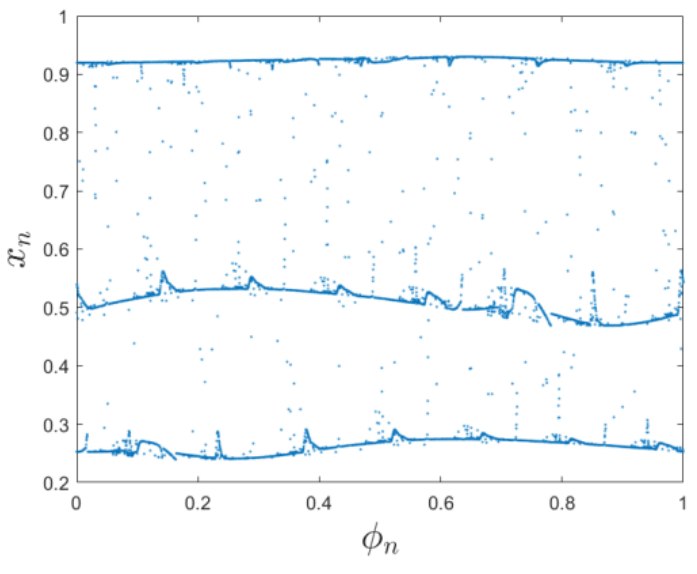

(d) $\omega_{19}=4181 / 6765$

Figure 13: For $r=1.95$, phase sensitivity functions. (a) $a=3.414, \varepsilon=0.1$, (b) $a=3.7028, \varepsilon=0.017$. Rational approximations. (c) $a=3.414, \varepsilon=0.1$, (d) $a=3.7028, \varepsilon=0.017$.

the control parameter. For $a \in[3,3.8]$, two different bifurcation diagrams are obtained by taking initial values $\left(x_{0}, y_{0}\right)=(0.1,0.3)$ and $\left(x_{0}, y_{0}\right)=(0.42,0.3)$, as shown in Figs. $17(\mathrm{a})$ and (b) respectively. When the range of $a$ is $a \in[3.41,3.45]$, we get the local enlargements of bifurcation diagrams shown in Figs. 17(c) and (d). The chaotic property is analyzed by the Lyapunov exponent, as shown in Figs. 17(e) and (f). For $a \in[3.416,3.438]$, there are the coexisting attractors. The initial value of the blue attractor is $\left(x_{0}, y_{0}\right)=(0.1,0.3)$, and that of the red attractor is $\left(x_{0}, y_{0}\right)=(0.42,0.3)$.

The phase diagrams with parameters (2) are shown in Fig. 18. For $a=3.41$, there is a $2 \mathrm{~T}$ quasiperiodic orbit (blue orbit) in the system, as shown in Fig. 18(a). When $a$ is increased to 3.43 , another $4 \mathrm{~T}$ quasiperiodic attractor (red trajectory) appears, and there is the coexistence of a $2 \mathrm{~T}$ quasiperiodic attractor and a $4 \mathrm{~T}$ one, as shown in Fig. 18(b). When $a$ is increased to 3.4332, the $4 \mathrm{~T}$ quasiperiodic attractor evolves into a SNA with 4 bands by the type-III intermittency, and the Lyapunov exponent $\lambda_{x}$ is -0.0021 . There is the the coexistence of a $2 \mathrm{~T}$ quasiperiodic attractor and SNA with 4 bands, as shown in Fig. 18(c). When $a$ is increased to 3.437, there is the the coexistence of a $2 \mathrm{~T}$ quasiperiodic attractor and a chaotic attractor with 4 bands, the Lyapunov exponent $\lambda_{x}$ is 0.0595 (red attractor), as shown in Fig. 18(d). When $a$ is increased to 3.438, 


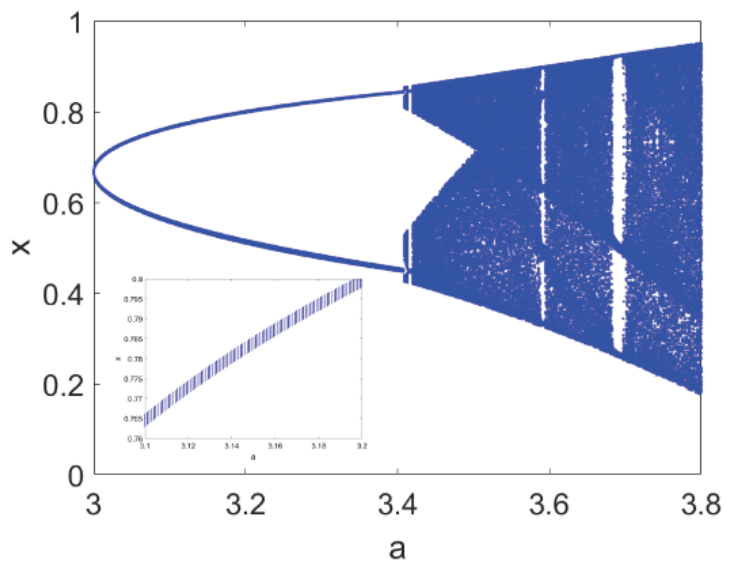

(a)

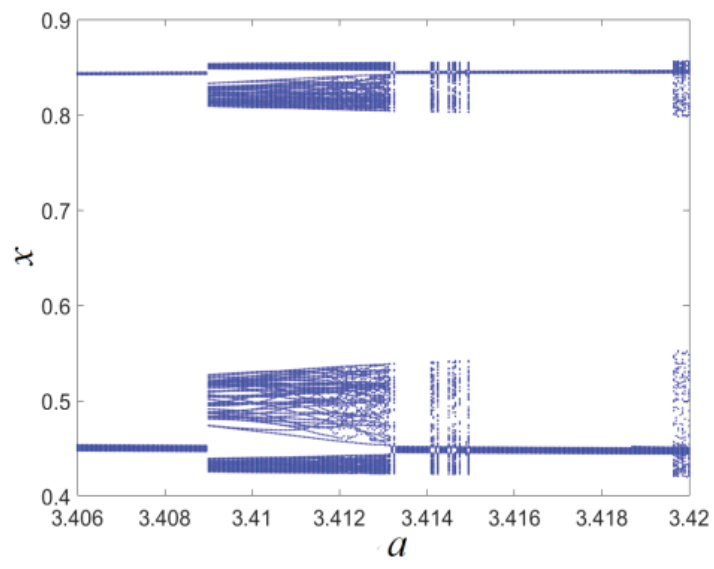

(c)

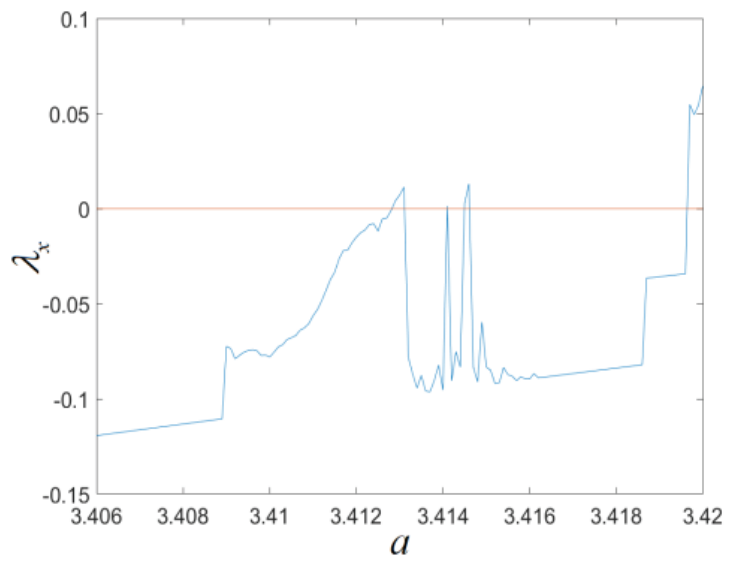

(e)

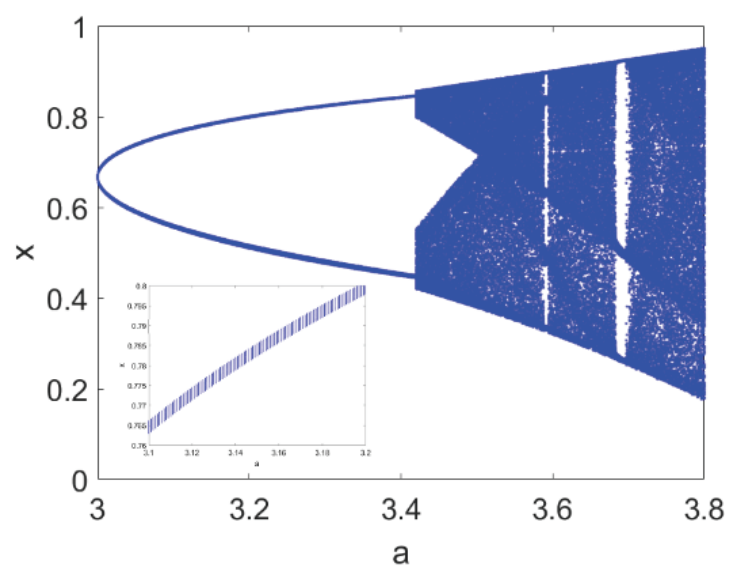

(b)

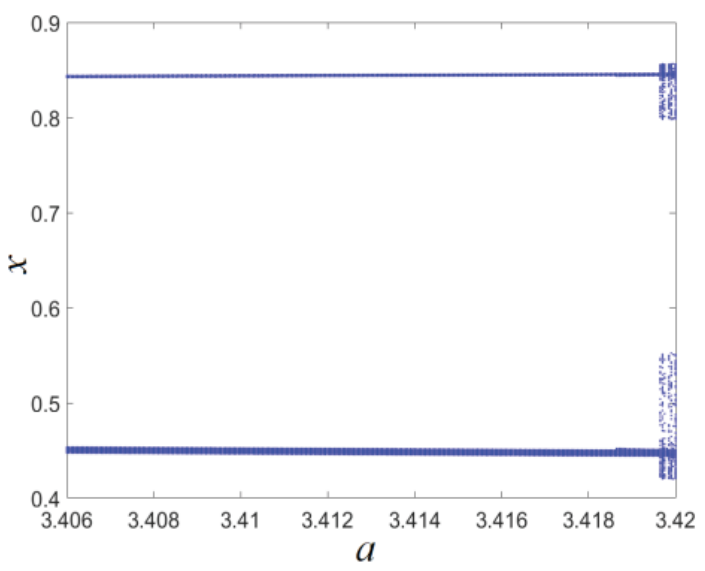

(d)

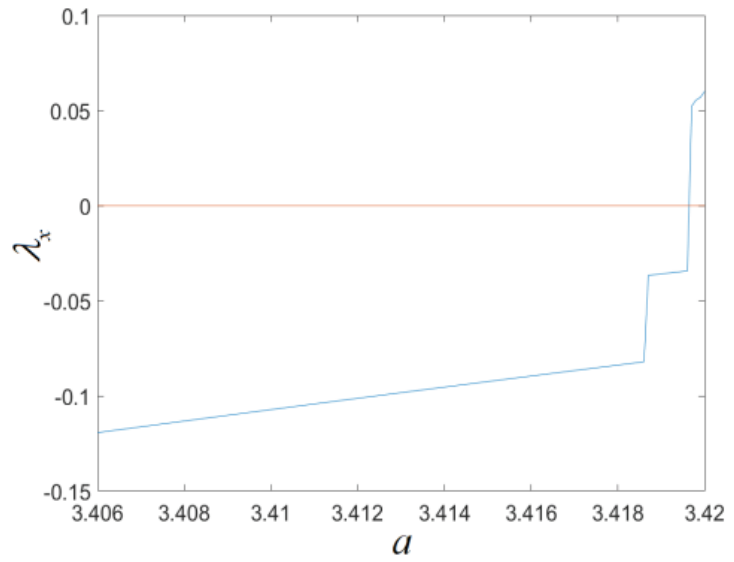

(f)

Figure 14: The bifurcation diagram with different initial values: $\varepsilon=0.006, r=1.9$. $(\mathrm{a})\left(x_{0}, y_{0}\right)=$ $(0.1,0.3)$, (b) $\left(x_{0}, y_{0}\right)=(0.42,0.3)$, (c) $\left(x_{0}, y_{0}\right)=(0.1,0.3)$. When $a \in[3.4063 .42]$, the Lyapunov exponent with $a$ varying. (d) $\left(x_{0}, y_{0}\right)=(0.42,0.3),(\mathrm{e}):\left(x_{0}, y_{0}\right)=(0.1,0.3),(\mathrm{f}):\left(x_{0}, y_{0}\right)=(0.42,0.3)$.

the blue $2 \mathrm{~T}$ quasiperiodic attractor disappears and only the chaotic attractor exists, the Lyapunov exponent $\lambda_{x}$ is 0.0655 , as shown in Fig. 18(e). For $a=3.44$, the red chaotic attractor with 4 bands disappears and the $2 \mathrm{~T}$ quasiperiodic attractor reappears, as shown 


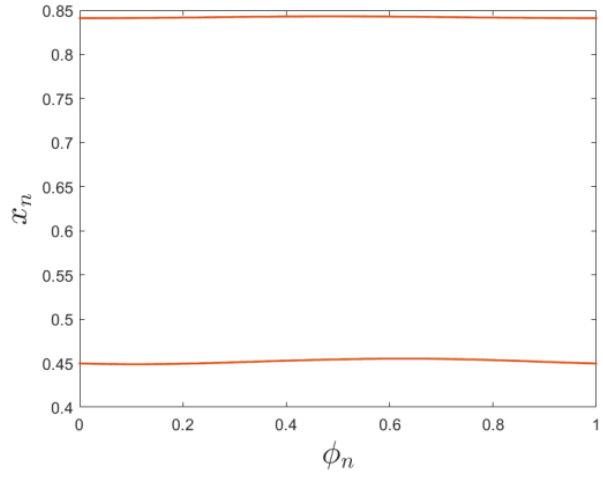

(a) $a=3.4$

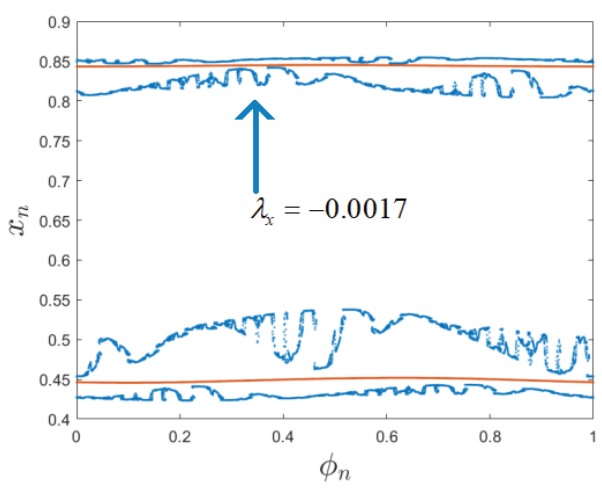

(c) $a=3.4127$

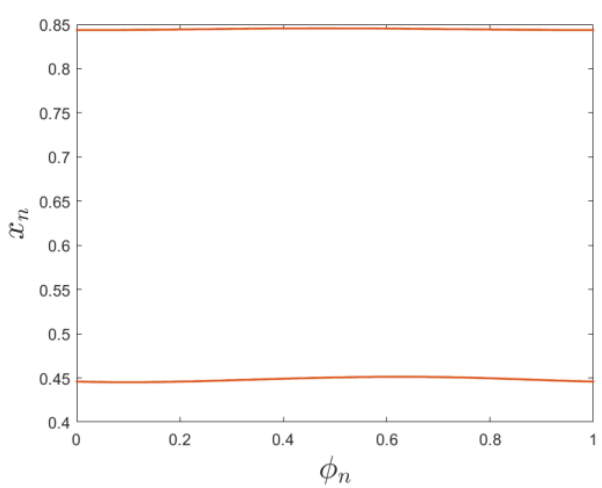

(e) $a=3.414$

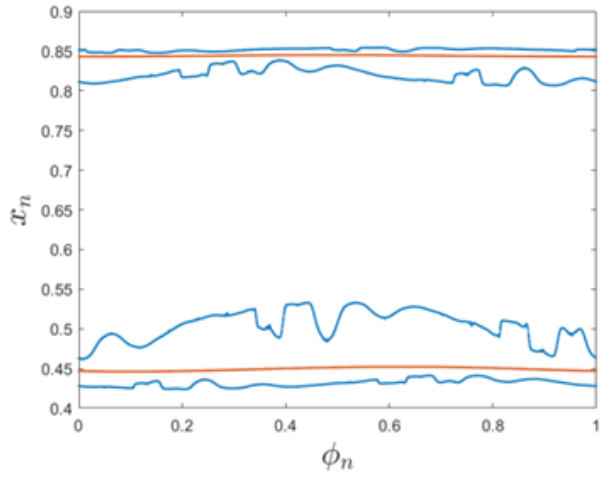

(b) $a=3.411$

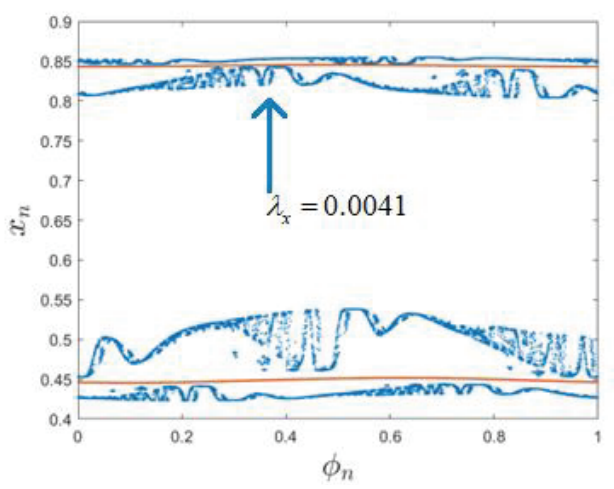

(d) $a=3.413$

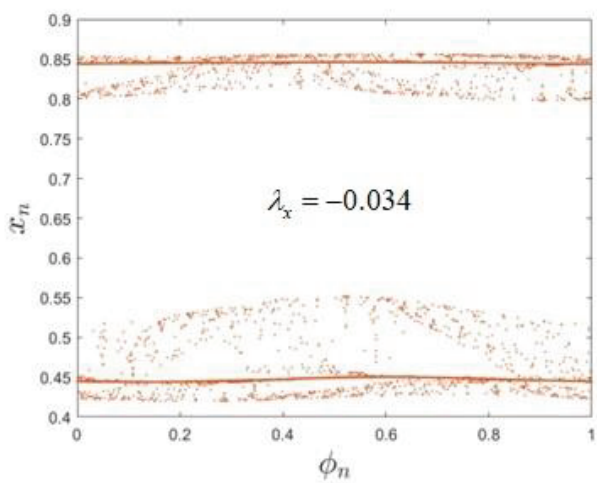

(f) $a=3.41964$

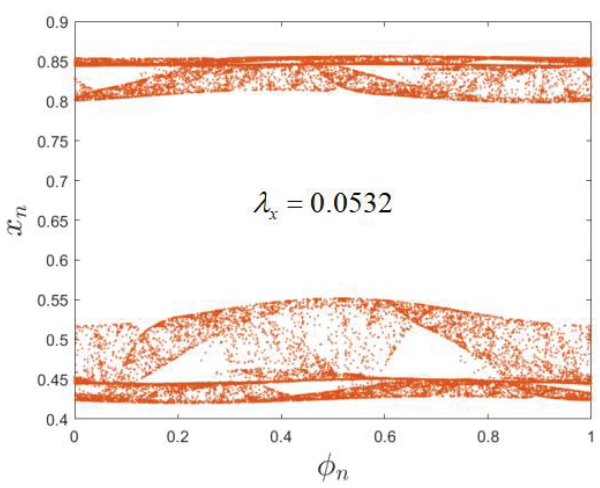

(g) $a=3.41965$

Figure 15: The coexistence of SNA and quasiperiodic attractor: $\varepsilon=0.006$ and $r=1.9$. 


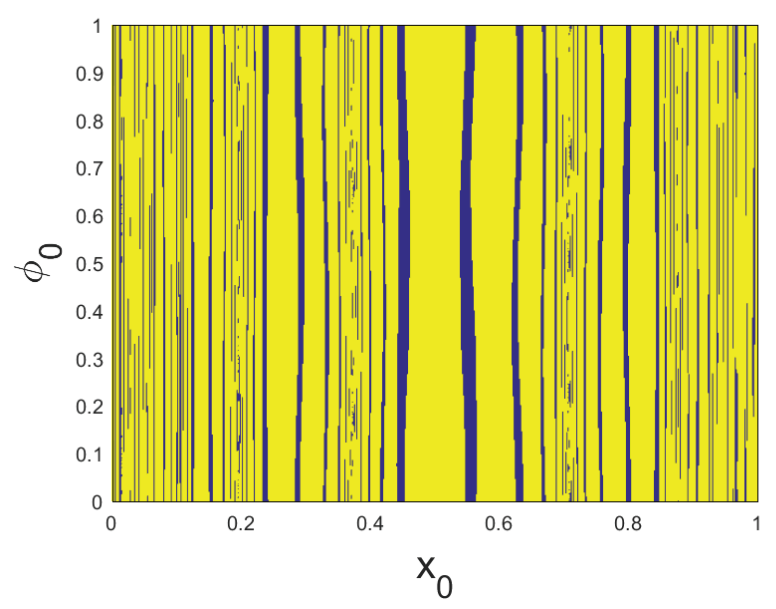

Figure 16: For $\varepsilon=0.006, r=1.9$, and $a=3.4127$, the basins of attraction.

in Fig. 18(f). Finally, for $a=3.448$, the $2 \mathrm{~T}$ quasiperiodic attractor turns directly into a chaotic attractor with 2 bands, as shown in Fig. 18(g). Taking $a=3.4332$, we obtain the basins of attraction corresponding to the attractors in Fig. 18(c), as shown in Fig. 19. If the initial value is in the yellow region, the orbit will eventually asymptote to the SNA with 4 bands (blue). If the initial value is in the blue region, the orbit finally asymptotes to the $2 \mathrm{~T}$ quasiperiodic attractor (red). From the bifurcation diagrams in Figs. 17(a) and (b), the crisis phenomenon can be observed. For $a \in[3.462,3.476]$, the evolution of the attractor is as follows: chaotic attractor with 4 bands $\rightarrow 6 \mathrm{~T}$ quasiperiodic attractor $\rightarrow$ chaotic attractor with 6 bands. For $a \in[3.59,3.603]$, the evolution of the attractor is as follows: chaotic attractor with 6 bands $\rightarrow 5 \mathrm{~T}$ quasiperiodic attractor $\rightarrow$ $10 \mathrm{~T}$ quasiperiodic attractor $\rightarrow$ chaotic attractor with 10 bands. For $a \in[3.687,3.717]$, the evolution of the attractor is as follows: chaotic attractor with 3 bands $\rightarrow$ SNA with 3 bands $\rightarrow$ 3T quasiperiodic attractor $\rightarrow$ SNA with 3 bands $\rightarrow$ chaotic attractor with 3 bands. The generated route of SNA is also caused by type-I intermittency.

\section{Conclusion}

In this paper, we consider a piecewise smooth system with quasiperiodic excitation. The global dynamic characteristics of the system are analyzed by two-parameter domains. There are three routes to SNAs in this piecewise system, including torus-doubling route, fractal route and intermittency route. The strange property of SNAs is characterized by phase sensitivity and rational approximations. For the nonsmooth systems with quasiperiodically forcing, a novel dynamical behavior that the SNA coexists with quasiperiodic attractors is uncovered. In such cases, basins of attraction are also obtained to undertand the global dynamics.

\section{Acknowledgments}

This work is supported by the National Natural Science Foundation of China (11672249, 11732014 and 11572263). 


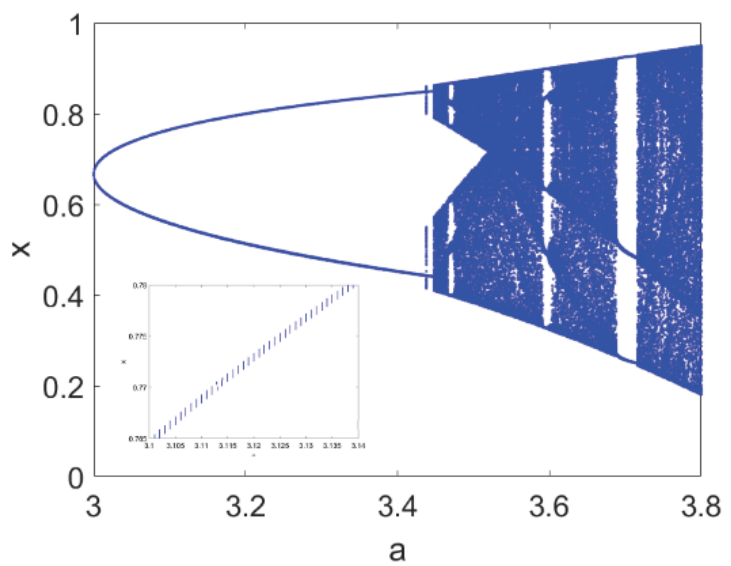

(a)

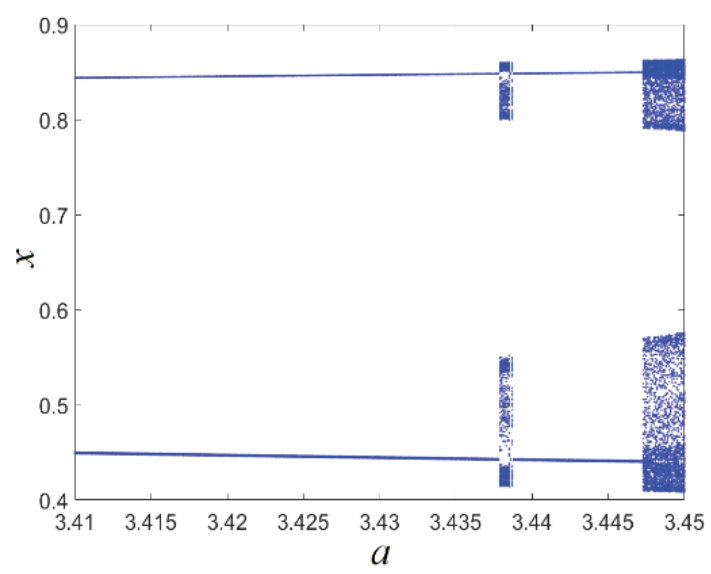

(c)

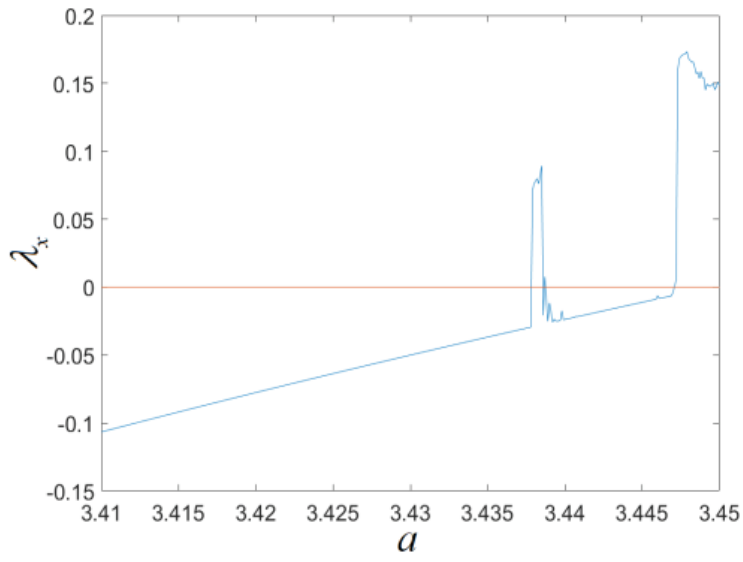

(e)

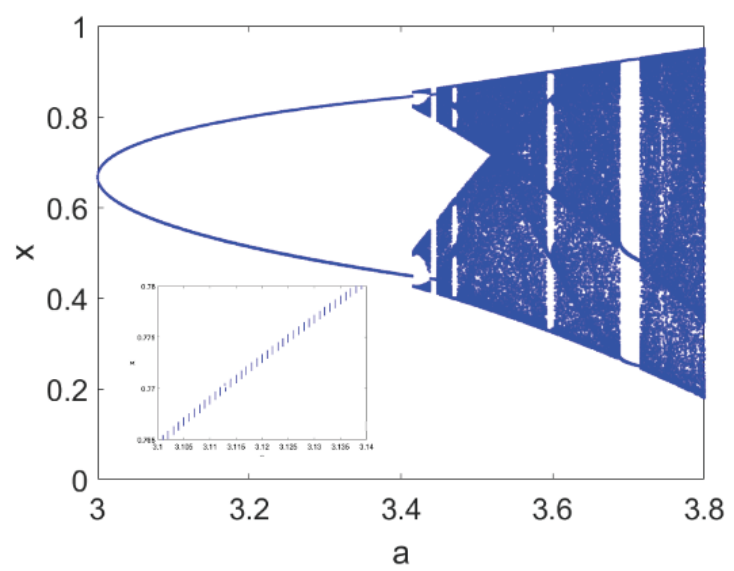

(b)

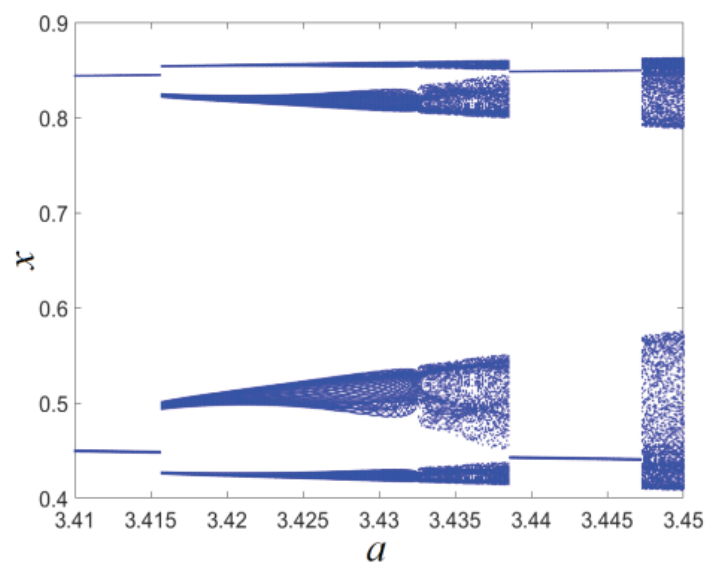

(d)

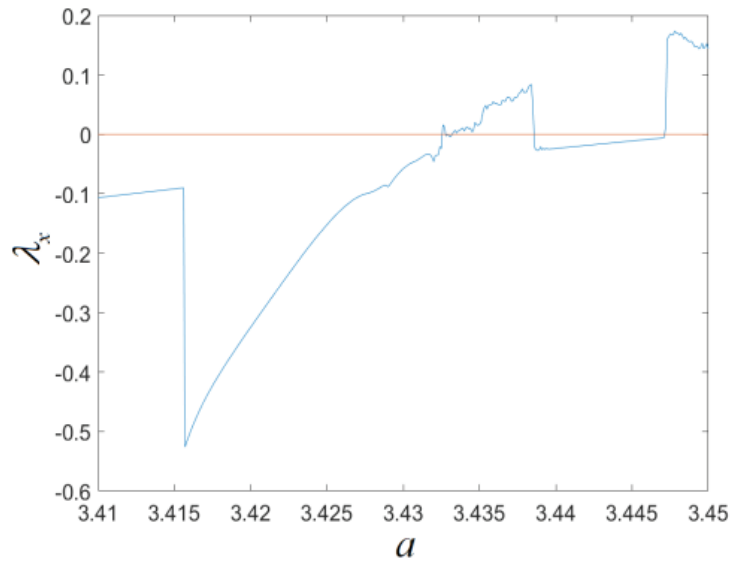

(f)

Figure 17: The bifurcation diagram with different initial values: $\varepsilon=0.0015, r=1.9312$. (a) $\left(x_{0}, y_{0}\right)=$ $(0.1,0.3)$, (b) $\left(x_{0}, y_{0}\right)=(0.42,0.3),(\mathrm{c})\left(x_{0}, y_{0}\right)=(0.1,0.3)$. When $a \in[3.4063 .42]$, the Lyapunov exponent with the variation of $a$. (d): $\left(x_{0}, y_{0}\right)=(0.42,0.3),(\mathrm{e})\left(x_{0}, y_{0}\right)=(0.1,0.3),(\mathrm{f})\left(x_{0}, y_{0}\right)=$ $(0.42,0.3)$.

\section{References}

[1] Grebogi, C., Ott, E., Pelikan, S., Yorke, J.A. Strange attractors that are not chaotic. Physica D Nonlinear Phenomena, 1984, 13(1): 261-268. 
[2] Sathish, A.M., Venkatesan, A., Lakshmanan, M. Strange nonchaotic attractors for computation. Physical Review E, 2018, 97(5): 052212.

[3] Zhang, Y. Wada basins of strange nonchaotic attractors in a quasiperiodically forced system. Physics Letters A, 2013, 377(18): 1269-1273.

[4] Ding, M., Grebogi, C., Ott, E. Dimensions of strange nonchaotic attractors. Physics Letters A, 1989, 137(4): 167-172.

[5] Zhang, X., Chen, G. Chaotic and non-chaotic strange attractors of a class of non-autonomous systems. Chaos: An Interdisciplinary Journal of Nonlinear Science, 2018, 28(2): 023102.

[6] Cabanas, A.M., Prez, L.M., Laroze, D. Strange non-chaotic attractors in spin valve systems. Journal of Magnetism and Magnetic Materials, 2018: S0304885317334959.

[7] Paul, A.M, Murali, K., Philominathan, P. Strange nonchaotic attractors in oscillators sharing nonlinearity, Chaos, Solitons and Fractals, 2019, 118: 83-93.

[8] Premraj, D., Suresh, K., Palanivel, J., Thamilmaran, K. Dynamic bifurcation and strange nonchaos in a two-frequency parametrically driven nonlinear oscillator. Communications in Nonlinear Science and Numerical Simulation, 2017, 50: 103-114.

[9] Yue, Y., Miao, P., Xie, J. Coexistence of strange nonchaotic attractors and a special mixed attractor caused by a new intermittency in a periodically driven vibro-impact system. Nonlinear Dynamics, 2017, 87(2): 1-21.

[10] Zhang, Y., Luo, G. Torus-doubling bifurcations and strange nonchaotic attractors in a vibro-impact system. Journal of Sound and Vibration, 2013, 332(21): 5462-5475.

[11] Wang, X., Zhan, M., Lai, C.H., Lai, Y.C. Strange nonchaotic attractors in random dynamical systems. Physical Review Letters, 2004, 92(7): 074-102.

[12] Ding, M., Grebogi, C., Ott, E. Evolution of attractors in quasiperiodically forced systems: From quasiperiodic to strange nonchaotic to chaotic. Physical Review A, 1989, 39(5):2593-2598.

[13] Heagy, J., Ditto, W.L. Dynamics of a two-frequency parametrically driven duffing oscillator. Journal of Nonlinear Science, 1991, 1(4): 423-455.

[14] Heagy, J.F., Hammel, S.M. The birth of strange nonchaotic attractors. Physica D, 1994, 70(1-2): 140-153.

[15] Nishikawa, T., Kaneko, K. Fractalization of torus revisited as a strange nonchaotic attractor, Phys. Physical Review E Statistical Physics Plasmas Fluids and Related Interdisciplinary Topics, 1996, 39(54): 6114-6124.

[16] Kim, J.W., Kim, S.Y., Hunt, B., Ott, E. Fractal properties of robust strange nonchaotic attractors in maps of two or more dimensions. Physical Review E, 2003, 67(3): 036211.

[17] Hunt, B.R., Ott, E. Fractal Properties of Robust Strange Nonchaotic Attractors. Physical Review Letters, 2001, 87(25): 254101.

[18] PrasadM, A., MehraM, V., RamaswamyM, R. Intermittency Route to Strange Nonchaotic Attractors. Physical Review Letters, 1997, 79(21): 4127-4130.

[19] Venkatesan, A., Murali, K., Lakshmanan, M. Birth of strange nonchaotic attractors through type III intermittency. Physics Letters A, 1999, 259(3-4): 246-253.

[20] Kim, S.Y., Lim, W., Ott, E. Mechanism for the Intermittent Route to Strange Nonchaotic Attractors. Physical Review E, 2003, 67(5): 056203. 
[21] Osinga, H.M., Feudel, U. Boundary crisis in quasiperiodically forced systems. Physica D, 2000, 141(1-2): $54-64$.

[22] Witt, A., Feudel, U., Pikovsky, A. Birth of strange nonchaotic attractors due to interior crisis. Physica D, 1997, 109(1-2): 180-190.

[23] Lindner, J.F., Kohar, V., Kia, B., Hippke, M., Learned, J.G., Ditto, W.L. Strange Nonchaotic Stars. Physical Review Letters, 2015, 114(5): 054-101.

[24] Keller, G. A note on strange nonchaotic attractors, Fund. Math. 1996, 151: 139-148.

[25] Alsedà, L., Misiurewicz, M. Attractors for unimodal quasiperiodically forced maps. Journal of Difference Equations and Applications, 2008, 14(10-11): 1175-1196.

[26] Glendinning, P., Jäeger, T., Keller, G. How chaotic are strange nonchaotic attractors. Nonlinearity, 2006, 19(9): 2005-2022.

[27] Osinga, H., Wiersig, J., Glendinning, P., Feudel, U. Multistability and nonsmooth bifurcations in the quasiperiodically forced circle map. International Journal of Bifurcation and Chaos, 2001, 11(12), 3085-3105.

[28] Yin, S., Jinchen, J., Deng, S., Wen, G. Neimark-Sacker bifurcations near degenerate grazing point in a two degree-of-freedom impact oscillator. Journal of Computational and Nonlinear Dynamics. 2018, 13 (11): 111007-111007-8.

[29] Yin, S., Wen, G., Xu, H., Wu, X. Higher order zero time discontinuity mapping for analysis of degenerate grazing bifurcations of impacting oscillators. Journal of Sound and Vibration. 2018, 437: 209-222.

[30] Shen, Y., Zhang, Y. Mechanisms of strange nonchaotic attractors in a nonsmooth system with border-collision bifurcations. Nonlinear Dynamics, 2019, 96: 1405-1428.

[31] Senthilkumar, D.V., Srinivasan, K., Thamilmaran, K., Lakshmanan, M. Bubbling route to strange nonchaotic attractor in a nonlinear series LCR circuit with a nonsinusoidal force. Phys. Rev. E 2008, 78: 066211

[32] Suresh, K., Prasad, A., Thamilmaran, K. Birth of strange nonchaotic attractors through formation and merging of bubbles in a quasiperiodically forced Chuas oscillator. Physics Letters A, 2013, 377(8): 612-621.

[33] Li, G., Yue, Y., Xie, J., Grebogi, C. Strange nonchaotic attractors in nonsmooth dynamical system. Communications in Nonlinear Science and Numerical Simulation, 2019, 78: 104858.

[34] Shen, Y., Zhang, Y. Strange nonchaotic attractors in a quasiperiodically forced piecewise smooth system with Farey tree. Fractals, 2019, 27: 1950118.

[35] Feudel, U., Grebogi, C. Multistability and the control of complexity. Chaos: An Interdisciplinary Journal of Nonlinear Science, 1997, 7(4): 597-604.

[36] Day, R. Irregular growth cycles. The American Economic Review, 1982; 72(3): 406C414.

[37] Pikovsky, A.S., Feudel, U. Characterizing strange nonchaotic attractors. Chaos: An Interdisciplinary Journal of Nonlinear Science, 1995, 5(1): 253-260.

[38] Pikovsky, A., Feudel, U. Correlations and spectra of strange nonchaotic attractors. Journal of Physics A: Mathematical and General, 1994, 27(15): 5209-5219.

[39] Feudel, U., Pikovsky, A., Politi, A. Renormalization of correlations and spectra of a strange nonchaotic attractor. Journal of Physics A General Physics, 1999, 29(17): 5297-5311. 
[40] Zhang, Y., Kong, G., Yu, J. Critical curves and coexisting attractors in a quasiperiodically forced delayed system. Physics Letters A, 2009, 373(15): 1341-1345.

[41] Zhang, Y., Kong, G. Multifarious intertwined basin boundaries of strange nonchaotic attractors in a quasiperiodically forced system. Physics Letters A, 2009, 374(2): 208-213. 


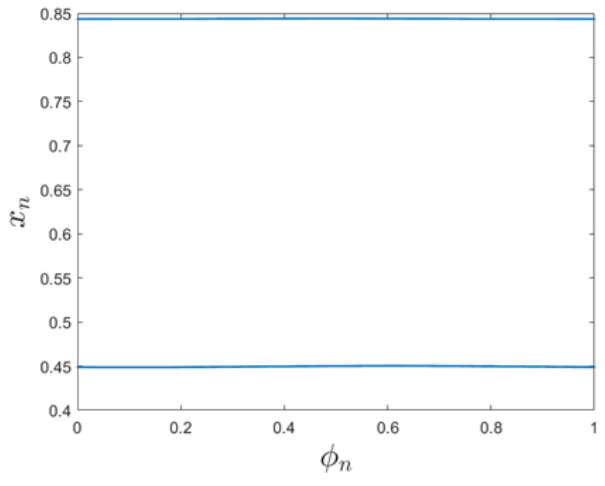

(a) $a=3.41$

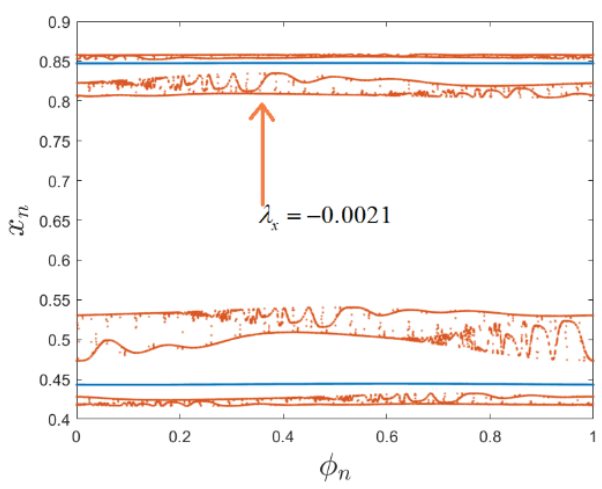

(c) $a=3.4332$

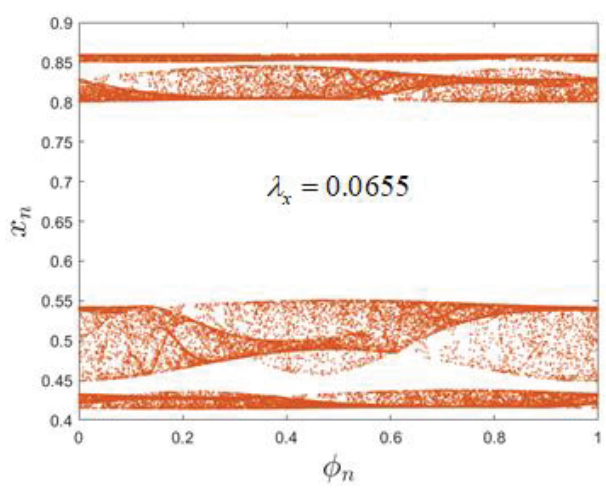

(e) $a=3.438$

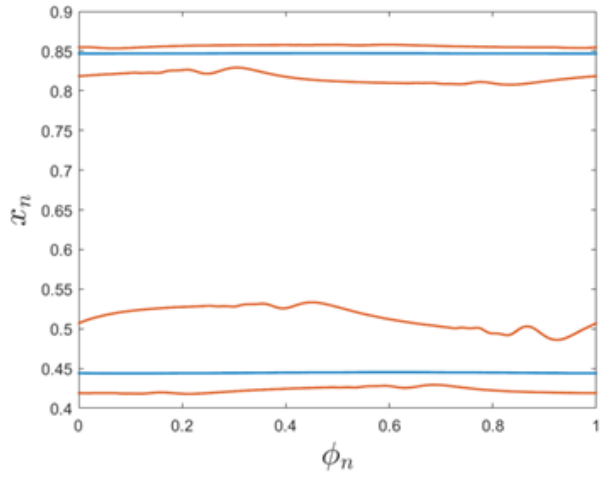

(b) $a=3.43$

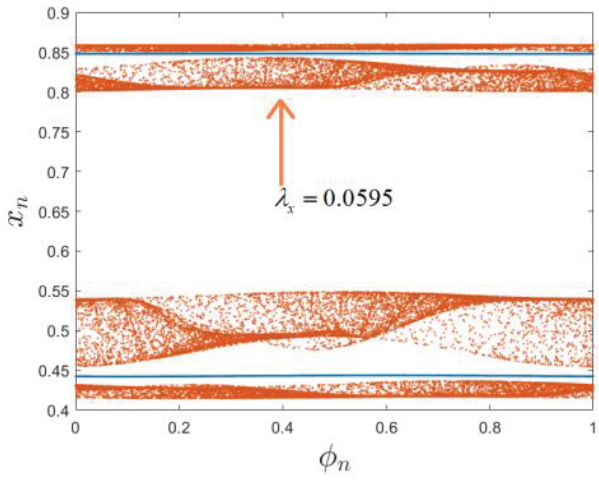

(d) $a=3.437$

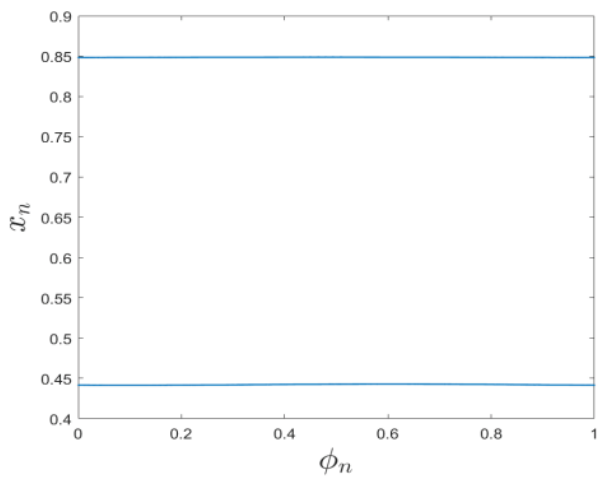

(f) $a=3.44$

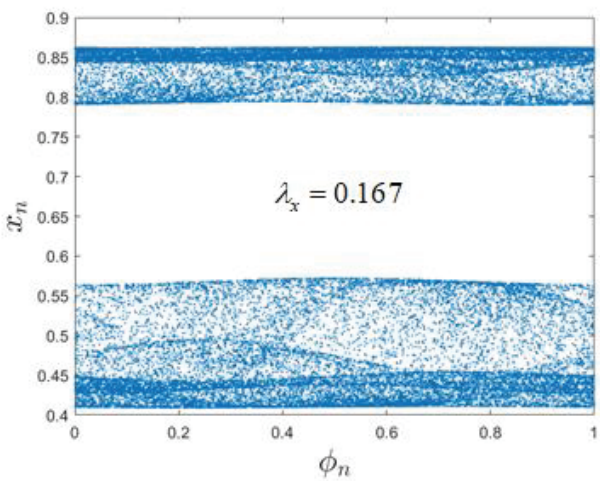

(g) $a=3.448$

Figure 18: The coexistence of SNA and quasiperiodic attractor: $\varepsilon=0.0015$ and $r=1.9312$. 


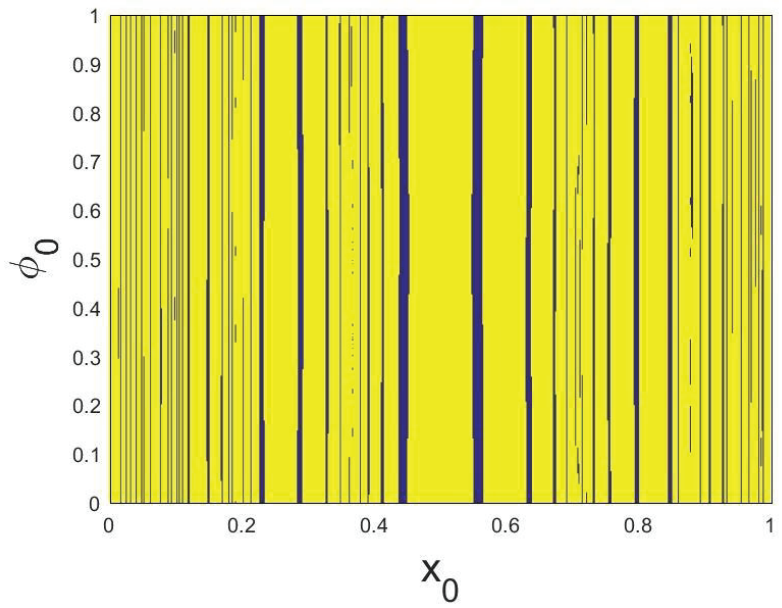

Figure 19: For $\varepsilon=0.0015, r=1.9312$ and $a=3.4332$, the basins of attraction. 